\title{
Resolving the X-Ray Obscuration in a Low-flux Observation of the Quasar PDS 456
}

\author{
J. N. Reeves ${ }^{1}(1)$, V. Braito ${ }^{1,2}$ (1) , E. Nardini ${ }^{3}$ (1) , F. Hamann ${ }^{4}$, G. Chartas ${ }^{5}$ (1) , A. P. Lobban ${ }^{6}$ (1), P. T. O’Brien ${ }^{7}$, and T. J. Turner ${ }^{8}$ (1) \\ ${ }^{1}$ Center for Space Science and Technology, University of Maryland Baltimore County, 1000 Hilltop Circle, Baltimore, MD 21250, USA; jreeves@umbc.edu \\ ${ }^{2}$ INAF, Osservatorio Astronomico di Brera, Via Bianchi 46 I-23807 Merate (LC), Italy \\ ${ }^{3}$ INAF-Osservatorio Astrofisico di Arcetri, Largo Enrico Fermi 5, I-50125 Firenze, Italy \\ ${ }_{5}^{4}$ Department of Physics \& Astronomy, University of California, Riverside, CA 92507, USA \\ ${ }^{5}$ Department of Physics \& Astronomy, College of Charleston, Charleston, SC 29424, USA \\ ${ }^{6}$ Astrophysics Group, School of Physical and Geographical Sciences, Keele University, Keele, Staffordshire, ST5 5BG, UK \\ ${ }^{7}$ Department of Physics and Astronomy, University of Leicester, University Road, Leicester LE1 7RH, UK \\ ${ }^{8}$ Department of Physics, University of Maryland Baltimore County, 1000 Hilltop Circle, Baltimore, MD 21250, USA \\ Received 2018 August 3; revised 2018 September 18; accepted 2018 September 18; published 2018 October 29
}

\begin{abstract}
Simultaneous XMM-Newton, NuSTAR, and HST observations, performed in 2017 March, of the nearby $(z=0.184)$ luminous quasar PDS 456 are presented. PDS 456 had a low X-ray flux compared to past observations, where the first of the two new XMM-Newton observations occurred during a pronounced dip in the X-ray light curve. The broadband X-ray spectrum is highly absorbed, attenuated by a soft X-ray absorber of column density $N_{\mathrm{H}}=6 \times 10^{22} \mathrm{~cm}^{-2}$. An increase in obscuration occurs during the dip, which may be due to an X-ray eclipse. In addition, the persistent, fast Fe $\mathrm{K}$ outflow is present, with velocity components of $-0.25 c$ and $-0.4 c$. The soft absorber is less ionized $(\log \xi=3)$ compared to the iron $\mathrm{K}$ outflow $(\log \xi=5)$ and is outflowing with a velocity of approximately $-0.2 c$. A soft $\mathrm{X}$-ray excess is present below $1 \mathrm{keV}$ against the highly absorbed continuum and can be attributed to the re-emission from a wide-angle wind. The complex X-ray absorption present in PDS 456 suggests that the wind is inhomogeneous, whereby the soft X-ray absorber originates from denser clumps or filaments that may form farther out along the outflow. In contrast to the X-ray observations, the simultaneous UV spectrum of PDS 456 is largely unabsorbed, where only a very weak broad absorption trough is present blueward of Ly $\alpha$, compared to a past observation in 2000 when the trough was significantly stronger. The relative weakness of the UV absorption may be due to the soft X-ray absorber being too highly ionized and almost transparent in the UV band.
\end{abstract}

Key words: black hole physics - galaxies: active - quasars: individual (PDS 456) - X-rays: galaxies

\section{Introduction}

Since their initial detection more than a decade ago (Chartas et al. 2002, 2003; Pounds et al. 2003; Reeves et al. 2003), the presence of ultrafast outflows has been found to be increasingly common in active galactic nuclei (AGNs). They were discovered through the signature of highly blueshifted absorption lines in the $\mathrm{Fe} \mathrm{K}$ band in the X-ray spectra of AGNs (Tombesi et al. 2010; Gofford et al. 2013). Given the large velocities $(v \sim 0.05 c-0.3 c)$ and column densities $\left(\sim 10^{23} \mathrm{~cm}^{-2}\right.$ or higher) of these systems, their mass outflow rate can be comparable to the quasar accretion rate (up to several solar masses per year), while the mechanical power of the outflows can reach a significant fraction of the AGN bolometric luminosity (Pounds \& Reeves 2009; Tombesi et al. 2013; Gofford et al. 2015; Nardini et al. 2015). At high redshifts, such AGN winds could have provided the mechanical feedback that controlled both the formation of stellar bulges and simultaneously self-regulated supermassive black hole growth, leading ultimately to the observed $M-\sigma$ relation for galaxies (Ferrarese $\&$ Merritt 2000; Gebhardt 2000). Indeed, evidence for the latter process has come from the discovery of massive, large $(\mathrm{kpc})$ scale molecular outflows (Feruglio et al. 2010; Veilleux et al. 2013; Cicone et al. 2014), which might be driven by the ultrafast outflows launched near the black hole (Feruglio et al. 2015, 2017; Tombesi et al. 2015; Fiore et al. 2017).

At a redshift of $z=0.184$ and with a total bolometric luminosity of $\sim 10^{47} \mathrm{erg} \mathrm{s}^{-1}$, PDS 456 is the most luminous QSO in the local universe (Torres et al. 1997; Simpson et al. 1999; Reeves et al. 2000) and is likely accreting near the
Eddington rate. It also hosts one of the best-studied examples of an X-ray ultrafast outflow. The fast wind in PDS 456 was first discovered in an XMM-Newton observation in 2001 (Reeves et al. 2003), through the detection of deep blueshifted absorption troughs, both above $7 \mathrm{keV}$ in the iron $\mathrm{K}$ band and above $1 \mathrm{keV}$ in the iron L-shell band. Since this initial detection, the persistence of the ultrafast outflow in PDS 456 has become established through over a decade's worth of X-ray observations (Reeves et al. 2009; Behar et al. 2010; Gofford et al. 2014; Reeves et al. 2014; Hagino et al. 2015; Nardini et al. 2015; Matzeu et al. 2016, 2017; Parker et al. 2018). It is also one of the fastest known winds, with the outflow velocity usually measured between $\sim 0.25 c$ and $0.3 c$ in all of the X-ray observations, with the exception of an additional faster $(\sim 0.4 c)$ relativistic component that was recently revealed in a highly absorbed NuSTAR observation in 2017 (Reeves et al. 2018a). The wide-angle emission from the wind, as measured through the P Cygni profile at iron K (Nardini et al. 2015), also implied that the wind subtends a large solid angle and that its mass outflow rate likely approaches the Eddington rate.

Both the X-ray continuum and the wind in PDS 456 are highly variable. The column density of the iron $\mathrm{K}$ absorption can vary by up to an order of magnitude over a few days (Gofford et al. 2014; Matzeu et al. 2016), implying that the fast wind is likely located within a few hundred gravitational radii of the black hole. Recently, a positive correlation was discovered between the outflow velocity and the X-ray luminosity (Matzeu et al. 2017), which suggests that radiation plays a role in accelerating the initial wind. The soft X-ray 
Table 1

X-Ray Observation Log

\begin{tabular}{lllll}
\hline \hline & NuSTAR & XMM OBS 1 & XMM OBS 2 & Swift \\
\hline Sequence & 60201020002 & 0780690201 & 0780690301 \\
Start date $^{a}$ & $2017 / 03 / 23$ & $2017 / 03 / 23$ & $2017 / 03 / 25$ & $2017 / 03 / 23$ \\
Start time $^{\mathrm{a}}$ & $05: 31: 09$ & $18: 56: 04$ & $05: 58: 10$ & $11: 30: 57$ \\
End date $^{\text {End time }}$ & $2017 / 03 / 26$ & $2017 / 03 / 24$ & $2017 / 03 / 26$ & $2017 / 04 / 10$ \\
Duration $^{\mathrm{b}}$ & $18: 36: 09$ & $17: 13: 40$ & $06: 28: 56$ & $08: 23: 57$ \\
\hline
\end{tabular}

Notes.

${ }^{\text {a }}$ Observation start and end time in UT.

${ }^{\mathrm{b}}$ Observation duration in ks.

spectrum of PDS 456 can become strongly absorbed, for instance, the Suzaku observations in 2013 caught PDS 456 with a highly absorbed spectrum $\left(N_{\mathrm{H}}>10^{23} \mathrm{~cm}^{-2}\right)$ at an extremely low flux (Matzeu et al. 2016). The soft X-ray absorption may arise from a clumpy component of the wind, which is capable of partially covering the X-ray source. Indeed, from an analysis of the XMM-Newton Reflection Grating Spectrometer (RGS) observations of PDS 456, Reeves et al. (2016) showed that the soft X-ray spectrum exhibits blueshifted absorption troughs, which may arise from a lower-ionization component of the wind. Recent observations of other AGNs are now also revealing soft X-ray components of ultrafast outflows, implying that the wind structure may be more complex than a simple homogeneous outflow (Pounds et al. 2003; Longinotti et al. 2015; Danehkar et al. 2018; Pinto et al. 2018; Reeves et al. 2018b).

In contrast to the well-studied X-ray wind in PDS 456, its UV spectrum has been poorly studied. An initial observation in 2000 with the Space Telescope Imaging Spectrograph (STIS) on board the Hubble Space Telescope (HST) revealed the presence of a broad absorption trough, blueshifted by $-0.06 c$ with respect to the broad Ly $\alpha$ emission line in the UV spectrum (O'Brien et al. 2005). Furthermore, several of the broad UV emission lines, such as from C IV, were blueshifted by several thousand kilometers per second, suggesting the presence of a broad-line region (BLR) scale wind component in PDS 456. A similar discovery was also recently made by Kriss et al. (2018b), who found a blueshifted Ly $\alpha$ trough associated with the ultrafast outflow in the QSO PG 1211+143. In PDS 456, Hamann et al. (2018) suggested that the Ly $\alpha$ trough may instead be associated with a highly blueshifted C IV absorption line, implying a UV outflow velocity of $-0.3 c$, similar to the wind velocity measured in X-rays. Since then, only one other UV spectrum of PDS 456 has been obtained, by HST COS in 2014, where the Ly $\alpha$ absorption trough appeared to be weaker compared to 2000 (Hamann et al. 2018). However, neither of the 2000 or 2014 UV spectra had simultaneous X-ray coverage, and thus the link between the X-ray and UV outflows in PDS 456 is poorly understood.

In this paper we present a detailed analysis of two recent simultaneous XMM-Newton and NUSTAR observations of PDS 456, in 2017 March. These observations caught PDS 456 in a low-flux state, similar to the 2013 Suzaku observations, where a prominent decrease in flux was observed during the first of these two observations. In Reeves et al. (2018a, hereafter Paper I), we analyzed the mean NuSTAR spectrum from the 2017 observations, which revealed a new ultrafast component of the $\mathrm{Fe} \mathrm{K}$ outflow, with an outflow velocity of $\sim 0.4 c$, in addition to the persistent $\sim 0.25 c$ wind. Here, we present the broadband X-ray spectral analysis, where the soft X-ray spectra are found to be strongly absorbed by a lower-ionization component of the wind. In addition, a simultaneous HST COS observation was performed during the X-ray observations, in order to better understand the link between the X-ray and UV wind in PDS 456.

The structure of this paper is as follows: In Section 2 we describe the new observations and X-ray variability of PDS 456, while in Section 3 we present the results of the broadband X-ray spectral analysis from the simultaneous 2017 March observations. In Section 4, we present the multiwavelength properties of PDS 456, including the simultaneous 2017 HST spectrum and the overall spectral energy distribution (SED). In Section 5, we discuss the properties of the soft X-ray absorber and its connection with the UV absorption in PDS 456.

\section{X-Ray Observations and Data Reduction}

PDS 456 was observed with NuSTAR (Harrison et al. 2013) from 2017 March 23 to 26, with a total duration of $305 \mathrm{ks}$. This coincided with two simultaneous XMM-Newton observations, hereafter OBS 1 and OBS 2, each of approximately $80 \mathrm{ks}$ duration. The XMM-Newton observations were taken over two consecutive satellite orbits and with both in Large Window mode for EPIC-pn (Strüder et al. 2001) and EPIC-MOS (Turner et al. 2001); see Table 1 for details of the observations. All data were processed using the NUSTARDAS v1.7.1, XMMNewton SAS v16.0, and HEASOFT v6.20 software. NuSTAR source spectra were extracted using a $50^{\prime \prime}$ circular region centered on the source and background from a $65^{\prime \prime}$ circular region clear from stray light. XMM-Newton EPIC-pn and spectra were extracted from single and double events, using a $30^{\prime \prime}$ radius source region and $2 \times 34^{\prime \prime}$ background regions on the same chip. Spectra from the RGS on board XMM-Newton (den Herder et al. 2001) for PDS 456 were extracted using the methods described in Reeves et al. (2016), where an analysis of the earlier archival XMM-Newton RGS spectra was presented. After checking for consistency, the RGS 1 and RGS 2 spectra were combined into a single RGS spectrum for each sequence, using the SAS task RGSCOMBINE. The XMM-Newton Optical Monitor (OM; Mason et al. 2001) data were acquired in "Imaging Mode" and were processed using the OMICHAIN task within SAS. Source coordinates were verified using the OMSOURCE task, and subsequent spectra were produced using OM2PHA. Data were acquired with all six OM filters: $V, B, U$, UVW1, UVM2 and UVW2. No source variability across the 
Table 2

List of X-Ray Exposures

\begin{tabular}{lcc}
\hline \hline Detector & OBS 1 & OBS 2 \\
\hline Exposures $^{\mathrm{a}}$ & 39.6 & 64.9 \\
XMM/pn & 62.2 & 84.9 \\
XMM/MOS & 72.0 & 88.0 \\
XMM/RGS & 38.2 & 47.2 \\
NuSTAR & & \\
Count rates & & $1.174 \pm 0.004$ \\
XMM/pn & $0.763 \pm 0.004$ & $0.635 \pm 0.003$ \\
XMM/MOS & $0.420 \pm 0.003$ & $0.058 \pm 0.001$ \\
XMM/RGS & $0.036 \pm 0.001$ & $0.096 \pm 0.002$ \\
NuSTAR & $0.072 \pm 0.002$ & \\
\hline
\end{tabular}

Notes.

${ }^{a}$ Net exposure times in ks after background screening and dead-time correction. Note that exposures are listed for an individual detector, e.g., MOS 1 or RGS 1 .

${ }^{\mathrm{b}}$ Net source count rates in $\mathrm{s}^{-1}$, after background subtraction. Count rates are for MOS 1+2, RGS 1+2, and NuSTAR FPMA+FPMB combined, respectively.

OM bandpass was detected within either observation, and so we proceeded to analyze the time-averaged photometry.

Periods of high background flaring (due to soft solar proton flares) were also removed from the two XMM-Newton observations. For the EPIC-pn detector, a threshold of 1 count $^{-1}$ over the full CCD field over the $10-12 \mathrm{keV}$ band was used to reject events corresponding to high particle background. For OBS 1, several background flares were present during the observation, and as a result the net exposures were reduced to $39.6,62.2$, and $72.0 \mathrm{ks}$ for the pn, MOS, and RGS, respectively, after removal of background flares and correcting for CCD dead time. Note that background flares are more severe for the pn CCD array than for the MOS CCDs, and as a result the net exposure time was lower. During OBS 2, the background level was stabler, and as a result, a higher net exposure time was obtained, of $64.9 \mathrm{ks}$ for EPIC-pn and $84.9 \mathrm{ks}$ for EPIC-MOS. The subsequent net exposure times and count rates are listed in Table 2; note that for the MOS and RGS the net count rates are for both detectors combined. After removal of the background flares, the background count rate is very low, contributing to only $2.6 \%$ and $1.2 \%$ of the total count rates over the $0.3-10 \mathrm{keV}$ band for the pn spectra of OBS 1 and OBS 2, respectively. Over the $3-10 \mathrm{keV}$ band the background rates are $5.8 \%$ and $2.9 \%$ of the total and have no effect on the net source spectrum over the iron $\mathrm{K}$ band.

Figure 1 shows the EPIC-pn X-ray light curves of the two $X M M$-Newton observations, superimposed on the light curve from the whole NuSTAR observation. The light curves from both satellites were extracted over the common 3-10 keV band and were binned into orbital (5814s) bins for the NuSTAR observation. PDS 456 shows pronounced X-ray variability during these observations. In particular, XMM-Newton OBS 1 coincided with a pronounced dip in the source count rate at $\sim 80 \mathrm{ks}$ into the NUSTAR observation. In addition to the NuSTAR observations, PDS 456 was monitored daily with Swift for 18 days during this campaign, with the Swift monitoring starting just after the beginning of the $2017 \mathrm{NuSTAR}$ observation. Figure 2 shows the Swift XRT light curve over the $0.3-10 \mathrm{keV}$ band, with the start and stop times of the
NUSTAR and the two XMM-Newton observations shown for comparison. From the Swift light curve, it is apparent that the $X M M-N e w t o n$ observations occurred during a relatively low period of X-ray flux during this campaign, which is especially the case for OBS 1 . Note that the details of the Swift monitoring will be presented in a subsequent paper, where, after the initial daily monitoring, PDS 456 was monitored weekly for a further 6 months, showing also pronounced long-term variability.

The total net exposure time of the whole NuSTAR observation is $157 \mathrm{ks}$. The mean NuSTAR spectrum was presented earlier in Paper I, where the presence of two velocity components of the fast outflow (with velocities $0.25 c$ and $0.43 c$ ) was revealed. In this paper, the main focus is on the broadband analysis between the XMM-Newton and NuSTAR observations, using simultaneous exposures in the soft and hard $\mathrm{X}$-ray bands. As a result of the pronounced X-ray variability, spectra from the NuSTAR FPMA and FPMB detectors were extracted only during the time intervals corresponding to the start and stop times of the XMM-Newton OBS 1 and OBS 2 exposures (see Table 1), so that the simultaneous broadband XMM-Newton and NUSTAR spectra could be analyzed for each sequence. Subsequently, the net NuSTAR exposures obtained for each sequence were 38.2 and $47.2 \mathrm{ks}$ for the OBS 1 and OBS 2 intervals, respectively. The NUSTAR spectra exclude time periods through and immediately after the passage of the satellite through the South Atlantic Anomaly. Furthermore, as the spectra obtained for the FPMA and FPMB modules were consistent, these were combined into single $N U S T A R$ spectra coincident with each of the OBS 1 and OBS 2 XMM-Newton sequences. All spectra are binned to at least 50 counts per bin to allow the use of $\chi^{2}$ minimization. Outflow velocities are given in the rest frame of PDS 456 at $z=0.184$, after correcting for relativistic Doppler shifts along the line of sight. Note that fluxes are stated without correcting for either Galactic or intrinsic absorption, whereas luminosities are corrected for absorption and quoted in the rest frame. Errors are quoted at $90 \%$ confidence for one interesting parameter (or $\left.\Delta \chi^{2}=2.7\right)$.

\section{Broadband Spectral Analysis}

In this section, we concentrate on the broadband X-ray spectral analysis, using the simultaneous XMM-Newton EPIC-pn and NuSTAR spectra taken during the OBS 1 and OBS 2 intervals. In particular, this provides an opportunity to study the broadband X-ray spectrum of PDS 456 during an unusually low $\mathrm{X}$-ray flux observation. Indeed, the $2-10 \mathrm{keV}$ flux derived during OBS 1 was $1.8 \times 10^{-12} \mathrm{erg} \mathrm{cm}^{-2} \mathrm{~s}^{-1}$, compared to the range of $(2.4-7.4) \times 10^{-12} \mathrm{erg} \mathrm{cm}^{-2} \mathrm{~s}^{-1}$ observed in the previous joint XMM-Newton and NUSTAR observations (OBS A-E) in 2013-14 analyzed by Nardini et al. (2015) and Matzeu et al. (2017). The pn and NuSTAR FPMA+FPMB spectra were fitted jointly, using the $0.3-10 \mathrm{keV}$ band for the pn and the 3.5-30 keV band for NUSTAR; note that for the latter the spectra become background dominated above $30 \mathrm{keV}$, and data above this energy were excluded from the spectral analysis. A multiplicative constant of $1.05 \pm 0.03$ was included between the NuSTAR and pn spectra to allow for any difference in normalization through cross-calibration and was allowed to vary as a free parameter in the modeling. We included a component of Galactic absorption in the spectral fitting, using the TBABS model of Wilms et al. (2000), where for PDS 456 the column density is expected to be $N_{\mathrm{H}}=2.4 \times 10^{21} \mathrm{~cm}^{-2}$ based on $21 \mathrm{~cm}$ 


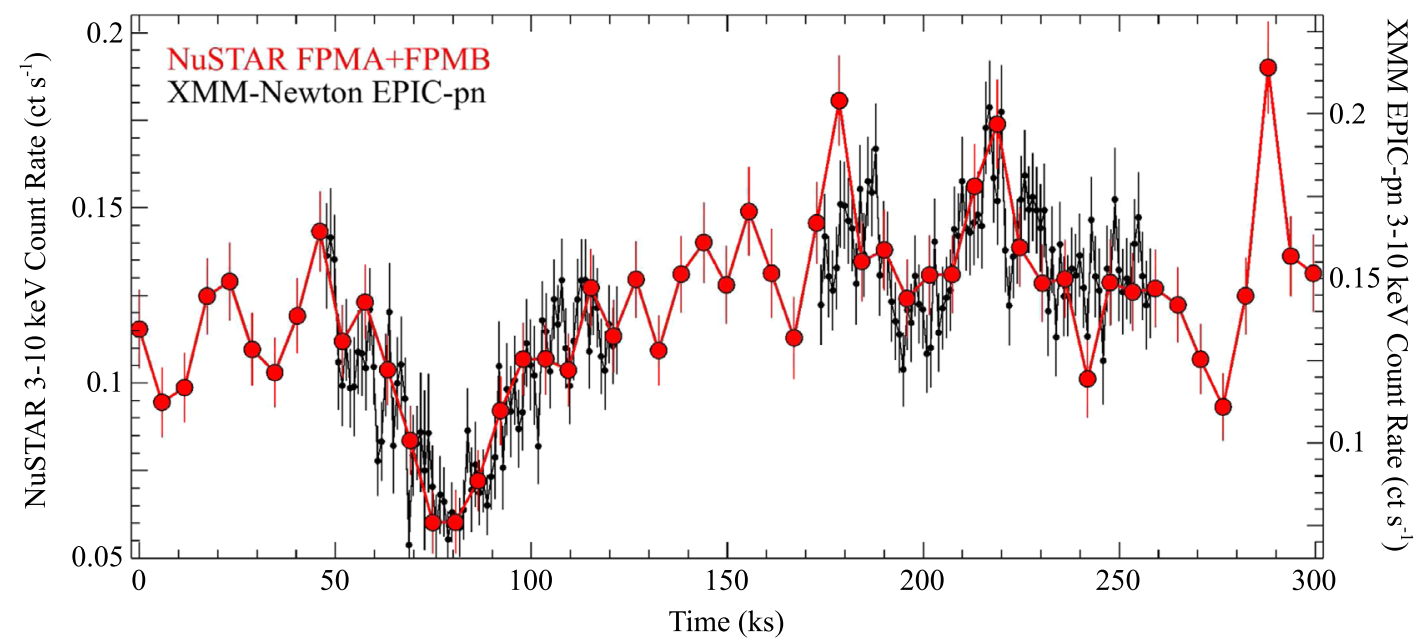

Figure 1. X-ray light curves of PDS 456 during the 2017 XMM-Newton and NuSTAR campaign. The background-subtracted NuSTAR (red circles) and XMM-Newton (black points) light curves were extracted over their common 3-10 keV band. Here the two XMM-Newton sequences correspond to OBS 1 and OBS 2, respectively. Note that the first XMM-Newton observation (OBS 1) coincided with a pronounced dip in the light curve.

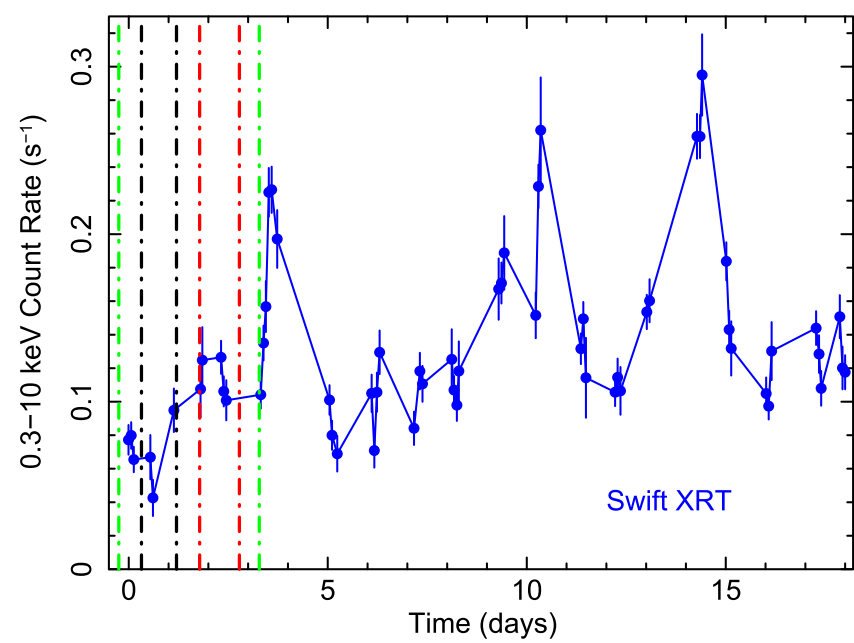

Figure 2. Swift daily monitoring of PDS 456, over the first 18 days of this campaign. The start and stop times of NUSTAR and XMM-Newton observations are denoted by dashed green (NUSTAR), black (XMM-Newton OBS 1), and red (XMM-Newton OBS 2) vertical lines (see Table 1 for details). Note the low flux of the pointed observations, especially XMM-Newton OBS 1, compared to later in the Swift campaign when pronounced X-ray flares are present.

measurements (Kalberla et al. 2005). Solar abundances of Grevesse \& Sauval (1998) were used throughout.

\subsection{The Overall Spectral Form}

Figure 3 shows the simultaneous pn and NUSTAR spectra from OBS 1 and OBS 2, compared to a $\Gamma=2.3$ power law fitted to the OBS 2 spectrum above $4 \mathrm{keV}$ for comparison and modified by Galactic absorption. This is close to the best-fit photon index found in the hard X-ray analysis in Paper I, as well as from historical spectra of PDS 456. A large deficit of counts is apparent against this simple power-law continuum in the soft X-ray band, with a minimum in the spectra occurring near $1.2 \mathrm{keV}$, blueward of the iron L-shell band. This is especially the case for OBS 1 , where the observation occurred during the pronounced dip in the light curve, and it appears at first glance that the spectrum is strongly obscured. Strong absorption is also present between 8 and $12 \mathrm{keV}$, blueward of the iron K-shell band, which again is very prominent in the
OBS 1 spectrum. Note that similar broadband residuals are also present from the EPIC-MOS spectra, in the form of absorption troughs in both the iron $\mathrm{L}$ and iron $\mathrm{K}$ bands. No neutral reflection component, associated with a distant reprocessor, has been included in the modeling, as there is no neutral iron $\mathrm{K} \alpha$ line or Compton hump present in the X-ray spectrum, consistent with what has been found from previous observations (Reeves et al. 2003, 2009; Nardini et al. 2015). The formal upper limit on the equivalent width of a narrow $6.4 \mathrm{keV}$ $\mathrm{K} \alpha$ line is $<45 \mathrm{eV}$, measured against the OBS 1 continuum.

We constructed an initial baseline model in order to account for the broadband spectra. This consisted of a power-law continuum, absorbed by a simple one-zone photoionized absorber, which is allowed to partially cover the X-ray source. Thus, the model is in the phenomenological form of

$$
\text { tbabs } \times\left[(1-f) \times \text { pow }+f \times \text { xstar }_{\text {pcov }} \times \text { pow }\right],
$$

where $f$ is the fraction of the power-law continuum (pow) that passes through the ionized absorber (denoted above by xstar ${ }_{\text {pcov }}$ ), and thus the fraction $1-f$ is unattenuated. Here TBABS represents the neutral Galactic photoelectric absorber, which absorbs the entire spectrum. A multiplicative grid of absorption models, generated by the XSTAR photoionization code (Kallman et al. 2004), was used to model the absorption. Here we adopt the same absorption model grids used in Nardini et al. (2015), where the mean optical to X-ray SED of PDS 456 was used as the input continuum, which has an ionizing (1-1000 ryd) luminosity of $L_{\text {ion }}=5 \times 10^{46} \mathrm{erg} \mathrm{s}^{-1}$. A turbulence velocity width of $15,000 \mathrm{~km} \mathrm{~s}^{-1}$ was used, which accounts for the velocity broadening of the $\mathrm{Fe} \mathrm{K}$ absorption features (Nardini et al. 2015; Reeves et al. 2018a).

The column density, outflow velocity, ionization parameter, and covering factor of the photoionized absorber were allowed to vary for each observation during the modeling, as was the normalization of the power-law continuum. The best-fit absorption parameters derived from this model consist of, for OBS 1, $N_{\mathrm{H}}=1.20_{-0.07}^{+0.04} \times 10^{23} \mathrm{~cm}^{-2}$, an ionization parameter of $\log \xi=3.42 \pm 0.02 \mathrm{erg} \mathrm{cm} \mathrm{s}^{-1}$, a covering fraction of $f=$ $0.77 \pm 0.02$, and an outflow velocity of $-0.239 c \pm 0.006 c$. For OBS 2, the spectrum is slightly less absorbed, with a lower 

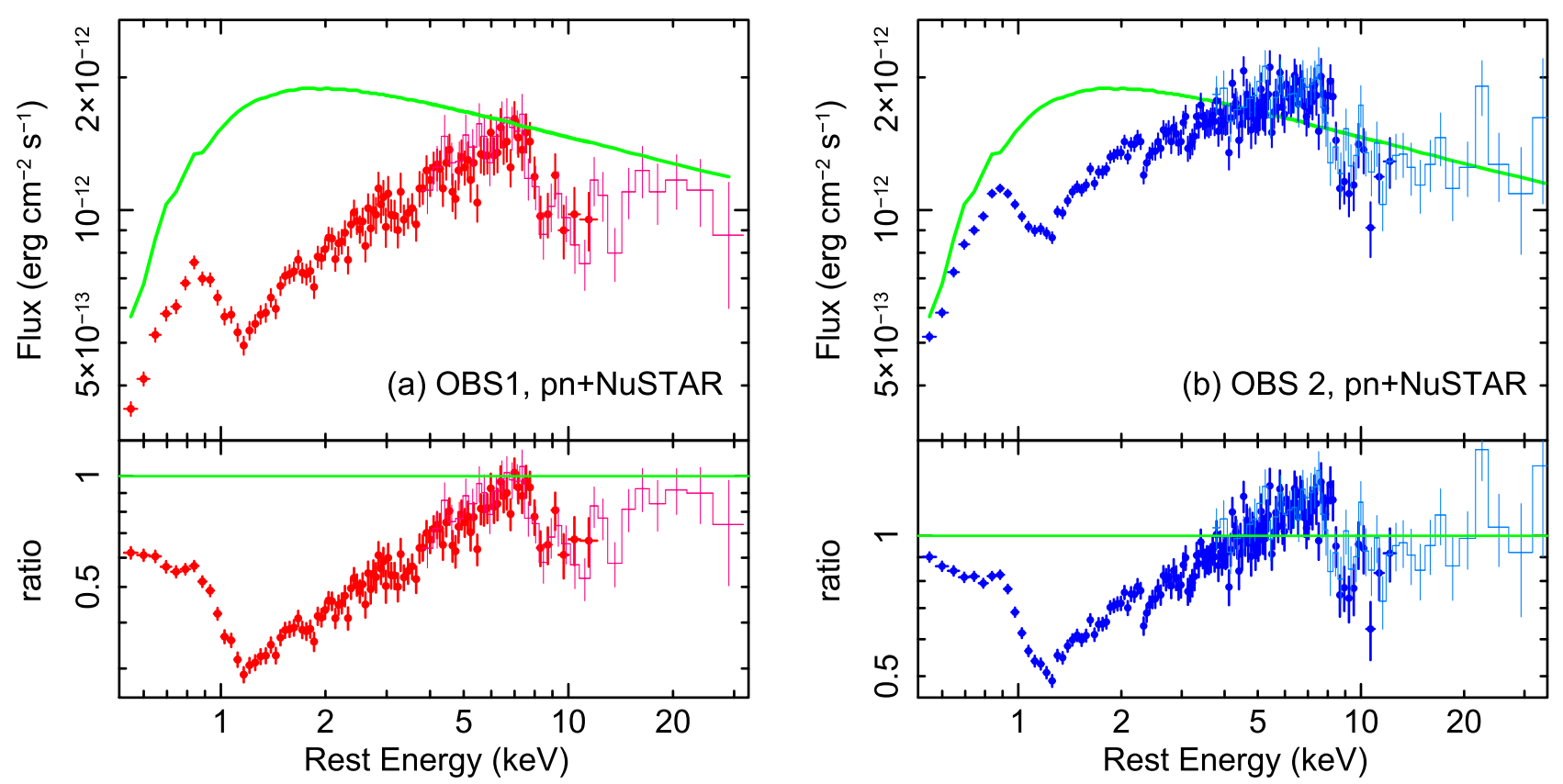

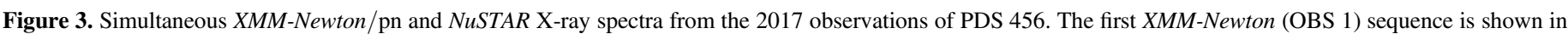

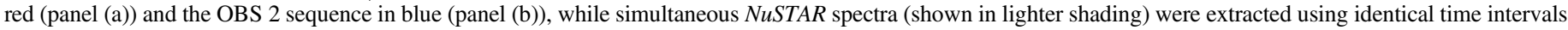

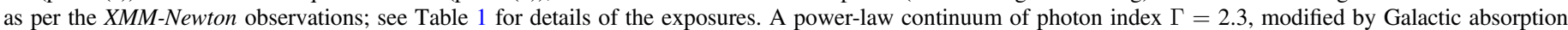

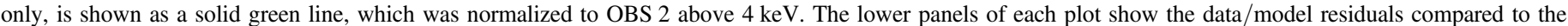

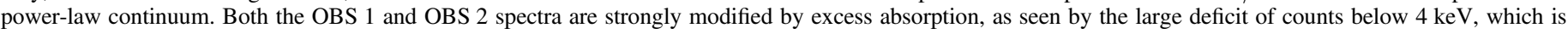

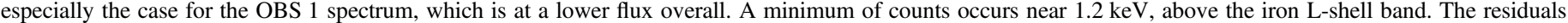

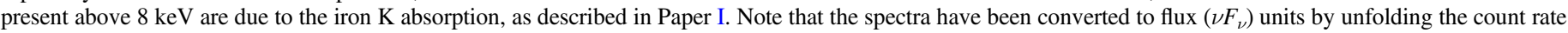
spectra against a $\Gamma=2$ continuum only.

column and covering fraction of $N_{\mathrm{H}}=0.94_{-0.05}^{+0.05} \times 10^{23} \mathrm{~cm}^{-2}$ and $f=0.70 \pm 0.02$, respectively, while the ionization parameter and outflow velocity are consistent between the two epochs. The continuum photon index is $\Gamma=2.43 \pm 0.03$, determined by the slope of the spectrum in the highest-energy band. Note that the high outflow velocity of the soft X-ray absorber is driven by the need to fit the broad absorption trough above $1 \mathrm{keV}$ with a blend of transitions from iron $\mathrm{L}$; the fit statistic worsened considerably from $\chi_{\nu}^{2}=1139 / 663$ to $\chi_{\nu}^{2}=1892 / 664$ if the outflow velocity was assumed to be zero, leaving large negative residuals between 1 and $1.5 \mathrm{keV}$. The velocity is also consistent with the velocity of the lowionization partial covering absorber derived by Matzeu et al. (2016), obtained during a similar low-flux observation in 2013 with Suzaku. It is also in agreement with what was inferred from the archival analysis of the previous XMM-Newton RGS spectra, from the presence of broad absorption troughs in the soft X-ray band (Reeves et al. 2016).

Nonetheless, despite the fact that the absorption model is able to reproduce the overall shape of the spectra through the large opacity and covering fraction of the absorber, the fit statistic is very poor, with $\chi_{\nu}^{2}=1139 / 663$, which is rejected with a null hypothesis probability of $p=1.2 \times 10^{-27}$. Strong residuals are still apparent in the data, compared to this baseline absorption model, as is shown in Figure 4. in particular, a strong excess in emission is seen in both spectra between 0.8 and $0.9 \mathrm{keV}$, which can be parameterized by a broad Gaussian profile, with an equivalent width of $85 \pm 15 \mathrm{eV}$ and a velocity width of $\sigma_{\mathrm{v}}=14,000 \pm 5000 \mathrm{~km} \mathrm{~s}^{-1}$, at a centroid energy of $0.88 \pm 0.01 \mathrm{keV}$. Furthermore, part of the broad absorption trough is missed between 1 and $2 \mathrm{keV}$, while the absorber is not able to model the iron K-band absorption seen between 8 and

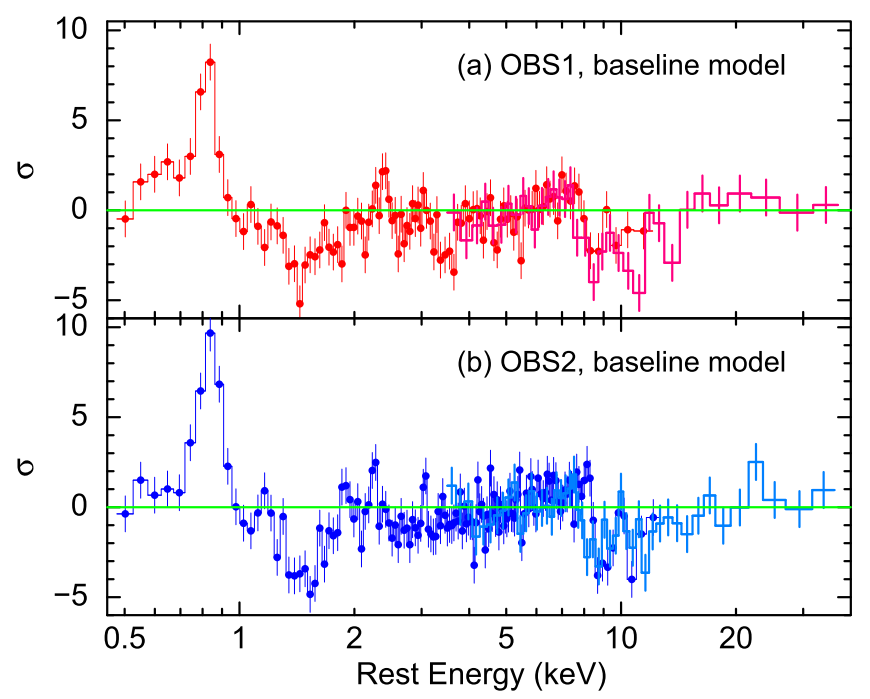

Figure 4. Residuals against the initial baseline partial covering model described in Section 3; panel (a) shows OBS 1, and panel (b) shows OBS 2. The residuals present in the iron $\mathrm{K}$ band are due to the two-component ultrafast outflow, with outflow velocities of $-0.25 c$ and $-0.43 c$ as described in Paper I. After modeling the spectra with the baseline model, strong residuals are still present in the soft X-ray band. An excess of counts is observed near $0.8 \mathrm{keV}$, while additional absorption is present above $1 \mathrm{keV}$; these remaining features may be associated with emission and absorption from L-shell iron originating from the wind, in addition to the iron K-band absorption above $8 \mathrm{keV}$.

$12 \mathrm{keV}$. This suggests that a single zone of absorption is not fully able to account for all of the features present in the data and that gas covering a wider range of ionization may be present. The relatively low ionization $(\log \xi=3.42 \pm$ $0.05 \mathrm{erg} \mathrm{cm} \mathrm{s}^{-1}$ ) of this absorber is driven primarily by the requirement to model the strong soft X-ray absorption present 
in the data, which likely originates from more moderately ionized iron (Fe XVII-XXIV) and from lighter elements, but which leaves the high-energy absorption from the $\mathrm{HE} / \mathrm{H}$-like $\mathrm{K}$-shell lines of iron unmodeled as a result.

\subsection{The Best-fit Broadband Model}

Thus, following the approach of Paper I, we added two additional (higher-ionization) zones of absorption fully covering the continuum, in order to account for the blueshifted absorption features seen in the iron K-band spectrum. This was modeled in Paper I, on the basis of the mean 2017 NuSTAR spectrum, by two outflowing absorbers, with outflow velocities of $-0.25 c$ and $-0.43 c$, originating from the blueshifted resonance $(1 s \rightarrow 2 p)$ absorption lines of $\mathrm{He} / \mathrm{H}$-like iron. The soft X-ray partial covering absorber is retained, as in the above model construction. In addition, we also include emission from photoionized gas, to account for the excess emission observed below $1 \mathrm{keV}$, as is seen in the residuals in Figure 4. Furthermore, in order to account for any velocity broadening of the emission, we followed the same methodology employed in Nardini et al. (2015) and used an additive XSTAR photoionized emission table generated for PDS 456, which is convolved with a Gaussian function. Thus, the form of this final model is

$$
\begin{aligned}
& \text { tbabs } \times \operatorname{xstar}_{\mathrm{FeK}} \times\left[(1-f) \times \text { pow }+f \times \text { xstar }_{\mathrm{pcov}}\right. \\
& \quad \times \text { pow }+ \text { Gauss } \otimes \text { emiss }],
\end{aligned}
$$

where xstar $_{\mathrm{Fe} \mathrm{K}}$ denotes the iron $\mathrm{K}$ absorbers and Gauss $\otimes$ emiss represents the photoionized emission convolved with a Gaussian profile. All the emission components are absorbed by a Galactic component of absorption, via the TBABS model, as above.

Overall this model produces an acceptable fit to the OBS 1 and OBS 2 spectra, with a fit statistic of $\chi^{2}=690 / 650$, significantly improved compared to the above model with just a single absorber zone and no additional emission. Figure 5 shows the resulting best fit to both data sets, where no strong residuals now remain compared to the model. The relative contributions of the emission components are shown as fitted to OBS 1; here the unabsorbed power law (green line) is absorbed only by the high-ionization (and low-opacity) Fe K absorber, while the magenta line shows the absorbed power law, which is additionally covered by the lower-ionization (high-opacity) absorber in the soft band. The much higher normalization of the absorbed power-law component, compared to the unabsorbed power law, can be seen by their relative contributions above $10 \mathrm{keV}$ in Figure 5, indicating that the covering fraction of the lower-ionization soft X-ray absorber is high, covering nearly $90 \%$ of the continuum in the case of OBS 1 (see Table 3 for details of the model parameters). The soft emission component (Figure 5, cyan line) makes a strong contribution toward the soft X-ray continuum and is able to model the excess line emission below $1 \mathrm{keV}$, as well as some broadened line emission in the iron $\mathrm{K}$ band. Its ionization is $\log \xi=$ $3.43 \pm 0.05$, similar to that of the partial covering absorber, and the emitter produces a blend of emission lines from L-shell $\mathrm{Fe}$ (Fe XVII-XIV), as well as from $\mathrm{He} / \mathrm{H}$-like $\mathrm{Ne}$ and $\mathrm{O}$. A large velocity broadening is required for this emission component, with a Gaussian width of $\sigma=0.80 \pm 0.05 \mathrm{keV}$ measured at $6 \mathrm{keV}$. As will be discussed in Section 3.5, this may represent the broadened emission component from a wide-angle wind.
Two outflowing absorption zones are required at $\mathrm{Fe} \mathrm{K}$ (denoted as $\operatorname{xstar}_{\mathrm{FeK}}$ above), one slower zone with $v_{\text {out }}=$ $-0.25 c \pm 0.02 c$ and the faster zone with $v_{\text {out }}=-0.43 c$ $\pm 0.02 c$; see Table 3 for details. Both outflowing zones at $\mathrm{Fe} \mathrm{K}$ are highly significant; their addition to the baseline model improved the fit by $\Delta \chi^{2}=-72.5$ and $\Delta \chi^{2}=-36.4$ for the slower and faster zones (hereafter zones 1a and 1b), respectively. As can be seen in Figure 5, these two iron K absorbers are able to account for the absorption observed between 8 and $12 \mathrm{keV}$ in both of the OBS 1 and OBS 2 spectra, with the faster zone reproducing the higher-energy absorption trough, as was described in depth in Paper I. The iron K zones are much more highly ionized than what is required to model the soft X-ray spectrum, with an ionization parameter of $\log \xi=4.9 \pm 0.1$ for the slower zone $1 \mathrm{a}$ and a lower limit on the ionization of $\log \xi>5.6$ for the faster zone $1 \mathrm{~b}$, which renders the latter component completely transparent in the soft $\mathrm{X}$-ray band.

In contrast, the soft X-ray absorber is of much lower ionization (Table 3, zone 2), with $\log \xi=3.05 \pm 0.05$, and reproduces the strong spectral curvature seen below $3 \mathrm{keV}$ in both spectra, as well as the broad absorption trough above $1 \mathrm{keV}$. Figure 6 shows the relative opacity (compared to a $\Gamma=2$ continuum) of this lower-ionization zone, with $\log \xi=3$ and $N_{\mathrm{H}}=5 \times 10^{22} \mathrm{~cm}^{-2}$, compared to the iron $\mathrm{K}$ zone absorber (zone 1a above), which has an ionization parameter two orders of magnitude higher with $\log \xi=5$ and $N_{\mathrm{H}}=2 \times$ $10^{23} \mathrm{~cm}^{-2}$. Despite its lower column, the low-ionization absorber produces much more opacity in the soft X-ray band, as well as a broad absorption trough at $\sim 1.2 \mathrm{keV}$, as is observed in both of the XMM-Newton spectra, which is primarily due to a blend of L-shell transitions of iron, as well as absorption from Ne IX-X. The outflow velocity of the soft $\mathrm{X}$-ray absorber is $-0.23 c \pm 0.02 c$, similar to the zone $1 \mathrm{a} \mathrm{Fe} \mathrm{K}$ absorber and also similar to that inferred in the analysis of the previous soft X-ray spectra of PDS 456 in Reeves et al. (2016). The high-ionization absorber produces mainly the strong resonance absorption in the Fe $\mathrm{K}$ band, through the blueshifted $1 s \rightarrow 2 p$ transitions of $\mathrm{He}$ and $\mathrm{H}$-like iron (Fe XXV and Fe XXVI). In contrast, this zone produces relatively little opacity in the soft band, compared to the lower-ionization absorber. Note that both zones do predict a strong blueshifted O VIII Ly $\alpha$ absorption line in the soft X-ray band, which, as we will show in Section 3.4, is detected in the higher-resolution XMM-Newton RGS spectrum.

\subsection{Spectral Variability}

Much of the spectral variability between the OBS 1 and OBS 2 spectra appears to be reproduced by the variability of the low-ionization partial covering absorber (zone 2), with the spectrum somewhat less absorbed in OBS 2 versus OBS 1 , although the former still requires substantial absorption. The column density and covering fraction of zone 2 for OBS 1 are $N_{\mathrm{H}}=6.6_{-0.5}^{+0.4} \times 10^{22} \mathrm{~cm}^{-2}$ and $f=0.87 \pm 0.03$, which then decrease to $N_{\mathrm{H}}=(5.4 \pm 0.4) \times 10^{22} \mathrm{~cm}^{-2}$ and $f=0.73 \pm$ 0.03 during OBS 2 . Note that this change is primarily driven by a change in the covering fraction: the fit statistic subsequently worsens by $\Delta \chi^{2}=52$ for $\Delta \nu=1$ if the covering fraction is forced to be identical between both data sets.

Indeed, the major difference between the two spectra is seen through the normalization of the unabsorbed power-law component; this more than doubles in flux from OBS 1 to OBS 2 from 


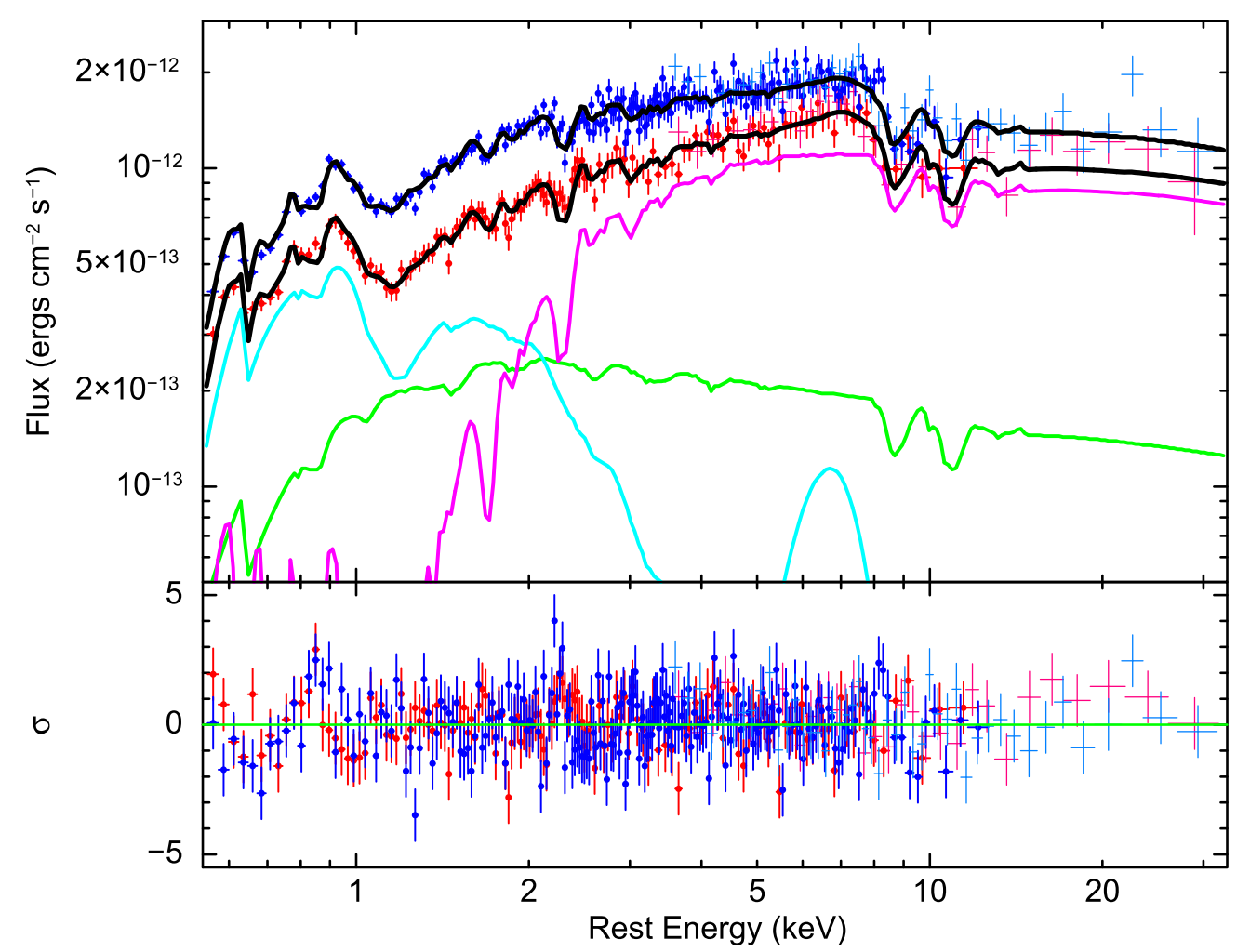

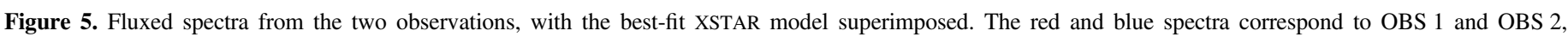

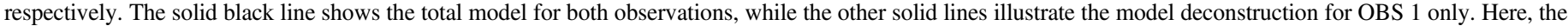

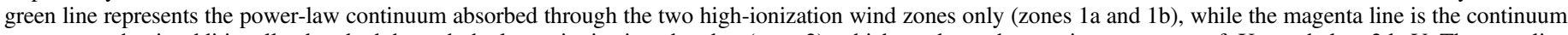

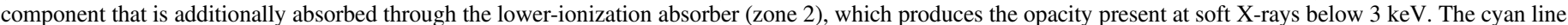

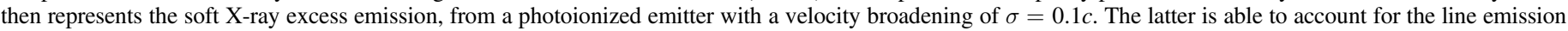
below $1 \mathrm{keV}$, as well as the broad emission at iron $\mathrm{K}$.

$N_{\text {UNABS }}=(2.0 \pm 0.3) \times 10^{-4}$ photons $\mathrm{cm}^{-2} \mathrm{~s}^{-1} \mathrm{keV}^{-1}$ (at $\left.1 \mathrm{keV}\right)$ to $N_{\text {UNABS }}=(5.0 \pm 0.4) \times 10^{-4}$ photons cm $\mathrm{cm}^{-1} \mathrm{keV}^{-1}$, while the normalization of the absorbed power-law component remains unchanged within the errors and thus was subsequently tied between the observations (see Table 3 for details). Alternatively, the fraction of the power-law continuum $(1-f)$ that emerges unattenuated by the soft X-ray absorber increases from $1-f=$ $0.13 \pm 0.03$ to $1-f=0.27 \pm 0.03$ from OBS 1 to OBS 2 .

\subsubsection{Variability around the Light-curve Dip}

To further test the change in obscuration around OBS 1 and whether the dip in the light curve is due to variable absorption, we isolated the pn spectrum of the dip in the light curve, centered within $\pm 10 \mathrm{ks}$ of the minimum in flux seen in Figure 1. For comparison, we then extracted the pn spectrum of the remaining OBS $1 \mathrm{pn}$ exposure, minus the portion around the dip. This resulted in a net exposure of $12 \mathrm{ks}$ in the dip and $28 \mathrm{ks}$ for the nondip portion of OBS 1 . The resulting spectra are shown in Figure 7 (left panel), where the dip spectrum is clearly fainter than the nondip portion, while the absorption trough just above $1 \mathrm{keV}$ increased in depth during the dip and the spectrum became harder.

We applied the above partial covering model to both spectra, varying only the covering fraction of the soft X-ray absorber (via the relative normalizations of the obscured and unobscured power-law components). The overall column density, ionization parameter, and outflow velocity of the soft X-ray absorber were also assumed to remain constant across OBS 1 . The highly ionized iron $\mathrm{K}$ absorption (zones $1 \mathrm{a}$ and $\mathrm{lb}$ ) was fixed to the mean values found for OBS 1 , as there is not enough signal in the short dip spectrum to determine whether the $\mathrm{Fe} \mathrm{K}$ profile varies or not. Similarly, the soft X-ray emission component was assumed to remain constant.

The variations between the dip and nondip spectra can be well modeled by a change in the covering fraction of the soft $\mathrm{X}$-ray absorber, and the overall fit statistic is good, with $\chi_{\nu}^{2}=$ $269.2 / 276$. Overall the soft X-ray $(0.5-2 \mathrm{keV})$ flux dropped by $50 \%$ from $F_{0.5-2.0 \mathrm{keV}}=9.1 \times 10^{-13} \mathrm{erg} \mathrm{cm}^{-2} \mathrm{~s}^{-1}$ for the nondip portion to $F_{0.5-2.0 \mathrm{keV}}=5.7 \times 10^{-13} \mathrm{erg} \mathrm{cm}^{-2} \mathrm{~s}^{-1}$ during the dip. The drop in flux can be accounted for by a decrease in normalization of the unobscured power law to $N_{\mathrm{UNABS}}<$ $3.6 \times 10^{-5}$ photons $\mathrm{cm}^{-2} \mathrm{~s}^{-1}$, formally consistent with zero. In comparison, the obscured power law dominates the spectrum, where $N_{\mathrm{ABS}}=(1.08 \pm 0.06) \times 10^{-3}$ photons $\mathrm{cm}^{-2} \mathrm{~s}^{-1}$; see Table 3 for details of the OBS 1 dip parameters. This implies that the covering fraction during the dip increased to $>97 \%$, consistent with a fully covering absorber. In contrast, the nondip spectrum is still absorbed, but with a lower covering fraction of $f=0.83 \pm 0.02$, consistent with the mean OBS 1 parameters. Note that the column density and ionization are consistent with the mean OBS 1 spectrum, with $N_{\mathrm{H}}=(6.2 \pm$ $0.5) \times 10^{22} \mathrm{~cm}^{-2}$ and $\log \xi=3.0 \pm 0.1$.

We also tested whether the increase in absorption could be due to a change in column rather than covering. In this case, the covering fraction (with $f=0.87 \pm 0.02$ ) was kept tied, while the soft X-ray column was allowed to vary and the overall continuum flux was allowed to adjust (via a constant multiplicative factor) to within $\pm 20 \%$ of the mean value. In this 
Table 3

Spectral Parameters for the 2017 XMM-Newton and NUSTAR Observations

\begin{tabular}{|c|c|c|c|}
\hline Parameter & OBS 1 & OBS 2 & OBS 1 Dip \\
\hline \multicolumn{4}{|c|}{ Fe K Absorber, Zone 1a } \\
\hline$N_{\mathrm{H}}^{\mathrm{a}}$ & $2.15 \pm 0.45$ & $1.50_{-0.25}^{+0.35}$ & $2.15^{\mathrm{b}}$ \\
\hline $\log \xi^{\mathrm{c}}$ & $4.9 \pm 0.1$ & $4.9^{\mathrm{d}}$ & $4.9^{\mathrm{b}}$ \\
\hline$v / c$ & $-0.247_{-0.012}^{+0.017}$ & $-0.259_{-0.013}^{+0.010}$ & $-0.247^{b}$ \\
\hline \multicolumn{4}{|c|}{ Fe K Absorber, Zone 1b } \\
\hline$N_{\mathrm{H}}{ }^{\mathrm{a}}$ & $7.3_{-2.2}^{+2.3}$ & $3.1_{-1.3}^{+1.5}$ & $7.3^{\mathrm{b}}$ \\
\hline $\log \xi^{\mathrm{c}}$ & $5.8_{-0.2}^{+1.2 \mathrm{e}}$ & $5.8^{\mathrm{d}}$ & $5.8^{\mathrm{b}}$ \\
\hline$v / c$ & $-0.43 \pm 0.02$ & $-0.43^{\mathrm{d}}$ & $0.43^{\mathrm{d}}$ \\
\hline \multicolumn{4}{|c|}{ Soft X-ray Absorber, Zone 2} \\
\hline$N_{\mathrm{H}}{ }^{\mathrm{a}}$ & $0.66_{-0.05}^{+0.04}$ & $0.54 \pm 0.04$ & $0.62 \pm 0.05$ \\
\hline $\log \xi^{\mathrm{c}}$ & $3.05 \pm 0.05$ & $3.05^{\mathrm{d}}$ & $3.0 \pm 0.1$ \\
\hline$v / c$ & $-0.23 \pm 0.01$ & $-0.23^{\mathrm{d}}$ & $-0.23^{b}$ \\
\hline$f^{f}$ & $0.87 \pm 0.03$ & $0.73 \pm 0.03$ & $>0.97$ \\
\hline \multicolumn{4}{|c|}{ Soft X-ray Emission } \\
\hline$N_{\mathrm{H}}^{\mathrm{a}}$ & $0.66^{\mathrm{b}}$ & $0.54^{\mathrm{b}}$ & $0.62^{\mathrm{d}}$ \\
\hline $\log \xi^{\mathrm{c}}$ & $3.43 \pm 0.05$ & $3.43^{\mathrm{d}}$ & $3.43^{\mathrm{b}}$ \\
\hline$\kappa_{\text {xstar }}{ }^{g}$ & $(0.68 \pm 0.06) \times 10^{-3}$ & $(0.82 \pm 0.07) \times 10^{-3}$ & $0.68^{\mathrm{b}}$ \\
\hline$\Omega / 4 \pi^{\mathrm{h}}$ & $0.72 \pm 0.07$ & $0.86 \pm 0.09$ & $\cdots$ \\
\hline \multicolumn{4}{|c|}{ Continuum } \\
\hline$\Gamma$ & $2.29 \pm 0.04$ & $2.29^{\mathrm{d}}$ & $2.29^{\mathrm{b}}$ \\
\hline$N_{\mathrm{ABS}}{ }^{\mathrm{i}}$ & $1.36 \pm 0.10$ & $1.36^{\mathrm{d}}$ & $1.08 \pm 0.06$ \\
\hline$N_{\text {UNABS }}{ }^{\mathrm{i}}$. & $0.20 \pm 0.03$ & $0.50 \pm 0.04$ & $<0.036$ \\
\hline$F_{2-10 \mathrm{keV}^{\mathrm{j}}}$ & 1.83 & 2.55 & 1.20 \\
\hline$L_{2-10 \mathrm{keV}^{\mathrm{k}}}$ & 2.71 & 3.44 & 1.84 \\
\hline$L_{7-30 \mathrm{keV}^{\mathrm{k}}}$ & 1.69 & 2.16 & $\cdots$ \\
\hline
\end{tabular}

Notes.

${ }^{\mathrm{a}}$ Units of column density $\times 10^{23} \mathrm{~cm}^{-2}$. Note that for the Fe K zone $1 \mathrm{~b}$, the $N_{\mathrm{H}}$ has been calculated for a fixed ionization parameter of $\log \xi=5.8$.

${ }^{\mathrm{b}}$ Denotes that the parameter is fixed.

${ }^{\mathrm{c}}$ Ionization parameter (where $\xi=L / n R^{2}$ ) in units of $\mathrm{erg} \mathrm{cm} \mathrm{s}^{-1}$.

${ }^{\mathrm{d}}$ Denotes that the parameter is tied between observations.

${ }^{\mathrm{e}}$ Denotes that the parameter error is pegged to the highest possible value in the model.

${ }^{\mathrm{f}}$ Covering fraction of absorber, where $f=1$ for a fully covering absorber.

${ }^{\mathrm{g}}$ Measured normalization of XSTAR emission component, where $\kappa_{\mathrm{xstar}}=$ $f_{\text {cov }} L_{38} / D_{\mathrm{kpc}}^{2}$ and $f_{\text {cov }}$ is the emitter covering fraction $(f=\Omega / 4 \pi), L_{38}$ is the $1-1000$ ryd ionizing luminosity in units of $10^{38} \mathrm{erg} \mathrm{s}^{-1}$, and $D_{\mathrm{kpc}}$ is the distance to PDS 456 in units of kpc.

${ }^{\mathrm{h}}$ Solid angle of the emission component, derived from the measured normalization of the emitter. See Section 3.5 for details.

${ }^{\mathrm{i}}$ Relative normalizations of the absorbed and unabsorbed power-law continuum components. Normalizations are in flux units of $\times 10^{-3}$ photons $\mathrm{cm}^{-2} \mathrm{~s}^{-1} \mathrm{keV}^{-1}$ at $1 \mathrm{keV}$.

${ }^{\mathrm{j}}$ Observed 2-10 keV flux, not corrected for absorption, in units of $\times 10^{-12} \mathrm{erg} \mathrm{cm}^{-2} \mathrm{~s}^{-1}$.

${ }^{\mathrm{k}}$ Intrinsic (absorption-corrected) $2-10 \mathrm{keV}$ (or 7-30 keV) luminosity, in units of $\times 10^{44} \mathrm{erg} \mathrm{s}^{-1}$.

case, the inferred column increased from $N_{\mathrm{H}}=(5.0 \pm 0.3) \times$ $10^{22} \mathrm{~cm}^{-2}$ to $N_{\mathrm{H}}=(9.1 \pm 0.7) \times 10^{22} \mathrm{~cm}^{-2}$ during the dip. However, the fit statistic is considerably worse, by $\Delta \chi^{2}=$ +44.4 (for $\Delta \nu=1$ ), compared to the variable covering case above, and the model is not fully able to reproduce the strong absorption trough above $1 \mathrm{keV}$ in the dip spectrum. Thus, the spectral variability could be due to an increase in the covering

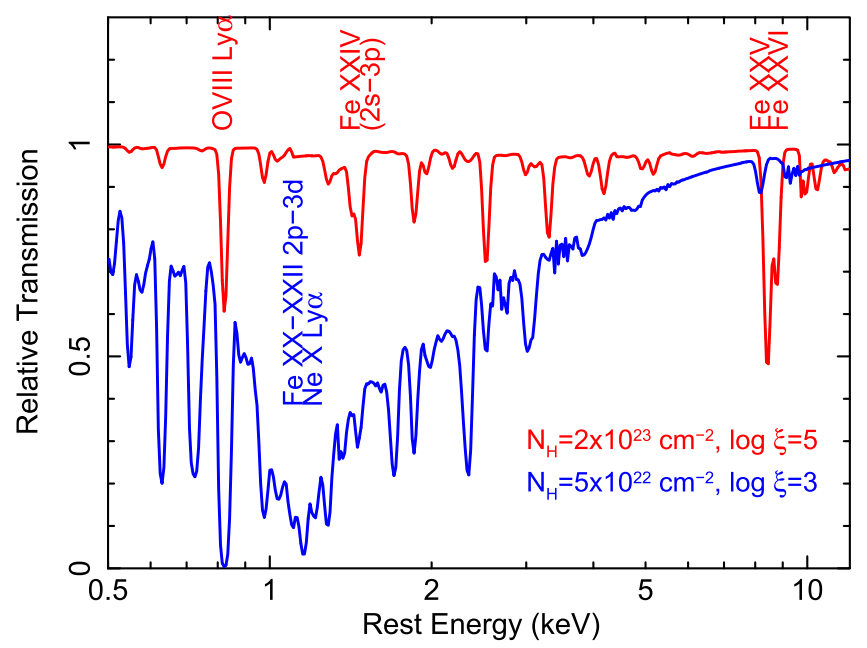

Figure 6. Relative transmission through two zones of absorption, representative of the absorption seen toward PDS 456 in these observations. Both zones are blueshifted by $-0.25 c$ and are corrected for Galactic absorption. The highionization absorber (shown in red) contributes toward the Fe $\mathrm{K}$ absorption, as seen through the $1 s \rightarrow 2 p$ lines of Fe XXV and Fe XXVI observed near $8.5 \mathrm{keV}$. The lower-ionization zone (shown in blue) has much greater opacity in the soft $\mathrm{X}$-ray band and in particular can reproduce the broad absorption trough seen near $1.2 \mathrm{keV}$ in these observations, as well as the strong spectral curvature. Both zones also produce blueshifted O VIII Ly $\alpha$ absorption near $0.8 \mathrm{keV}$, as is also detected in the RGS spectrum. The simulation also illustrates the wealth of wind features that may be observed in future X-ray calorimeter spectra, from $X A R M$ and Athena.

fraction of the soft X-ray absorber, reaching a maximum full covering during the flux minimum in OBS 1 , while the covering fraction is at its lowest during the brighter OBS 2 sequence.

To test the variability in a more model-independent way, we examined the NuSTAR count rate light curve across the dip portion of the observations, comparing the softer $3-5 \mathrm{keV}$ NuSTAR band with the continuum-dominated $20-40 \mathrm{keV}$ hard $\mathrm{X}$-ray band. Any absorption variability should preferentially affect the softer band more than the harder band, while if the dip is due to (colorless) changes in the power-law continuum, then both bands should be variable. Figure 7 (right panel) shows the light curves in these bands, over the first portion of the NUSTAR observation. The dip is clearly apparent in the softer $3-5 \mathrm{keV}$ band, occurring $80 \mathrm{ks}$ into the NUSTAR observation, consistent with Figure 1. However, no dip or pronounced variability is seen in the $20-40 \mathrm{keV}$ band at these times. Thus, the dip is primarily due to an increase in obscuration, rather than a simple decrease in the intrinsic power-law continuum, as the latter should also produce a decline in flux in the $20-40 \mathrm{keV}$ band.

\subsection{The Soft X-Ray Spectrum}

We now investigate the soft X-ray spectrum of PDS 456 in more detail. We first used the RGS grating spectra obtained from both XMM-Newton observations, over the 6-30 ̊ range. The signal-to-noise ratio is more limited in the individual RGS spectra of each sequence, and as they are not sensitive to any subtle changes in the absorption parameters, we subsequently combined these into a single time-averaged RGS spectrum from both observations. The resulting spectrum was then binned into wavelength bins of width $\Delta \lambda=0.1 \AA$, approximately the FWHM resolution of the RGS gratings. Note that the observed $0.5-2.0 \mathrm{keV}$ flux obtained from the RGS 

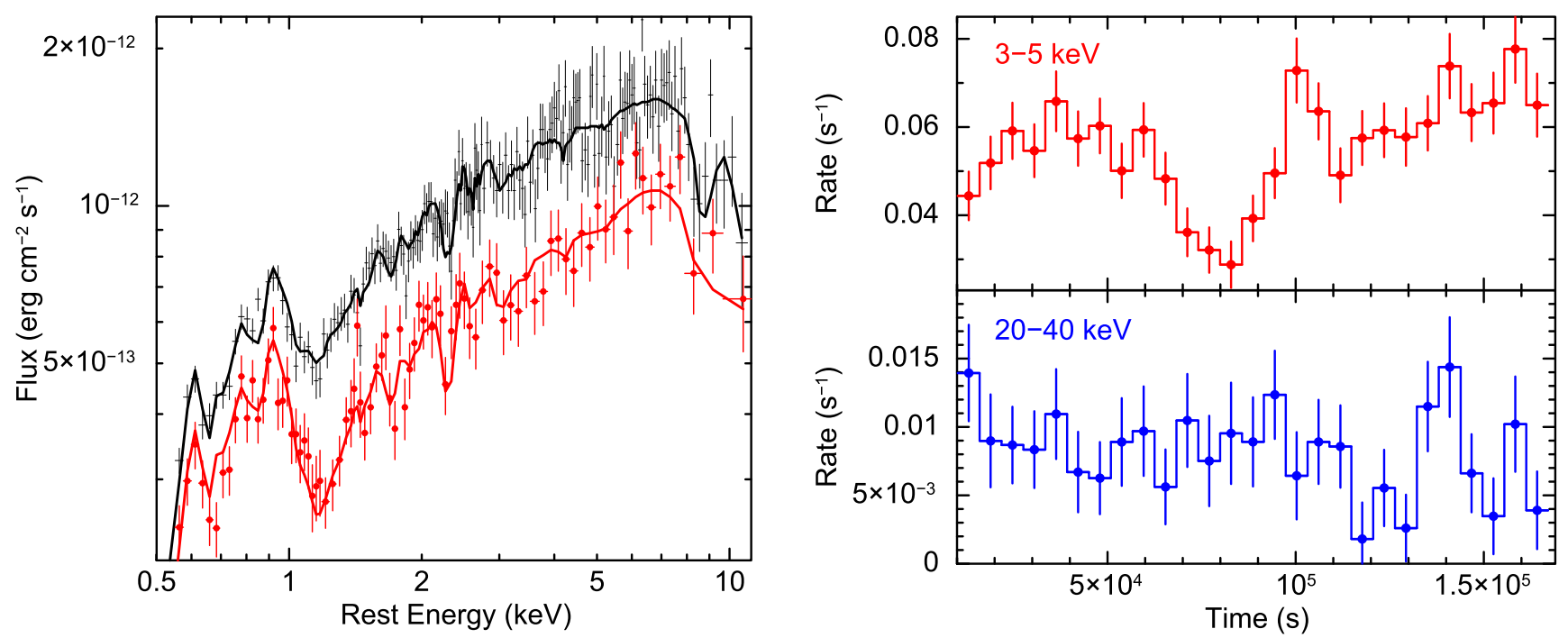

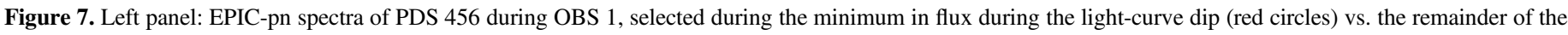

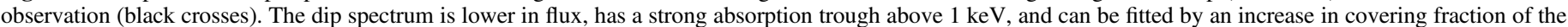

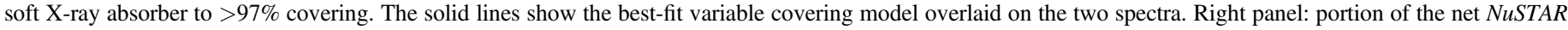

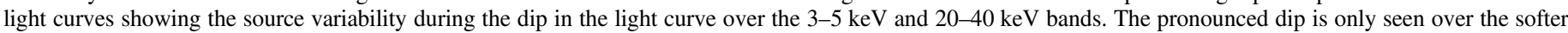

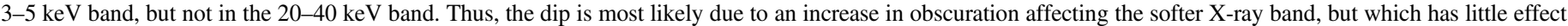
on the hard X-ray continuum from 20 to $40 \mathrm{keV}$.

from these observations is $1.0 \times 10^{-12} \mathrm{erg} \mathrm{cm}^{-2} \mathrm{~s}^{-1}$; this compares to the flux range of $F_{0.5-2 \mathrm{keV}}=1.6-4.3 \times$ $10^{-12} \mathrm{erg} \mathrm{cm}^{-2} \mathrm{~s}^{-1}$ for the previous archival XMM-Newton RGS observations from 2001 to 2014 (Reeves et al. 2016). The 2017 soft X-ray spectrum thus represents a historical low flux, where PDS 456 is highly absorbed and likely dominated by any reprocessed components associated with the wind.

A fit to a simple soft power law, of $\Gamma=2.3 \pm 0.1$, absorbed only by the neutral Galactic column, yields an extremely poor fit to the mean spectrum with $\chi_{\nu}^{2}=358 / 229$, rejected with a null hypothesis probability of $9.6 \times 10^{-8}$. Similar residuals are seen in the soft X-ray band, as per the EPIC-pn CCD spectrum, with a broad absorption trough between 10 and $12 \AA$ and an excess of emission between 12 and $17 \AA$. Thus, we adopt a similar continuum parameterization for the RGS as per the broadband spectrum above, where the power-law continuum is partially covered by the soft-band absorber (and where a fraction $1-f$ remains unattenuated). A broadened emission component is also included for the excess soft X-ray emission, as per the above broadband analysis. The photon index of the intrinsic continuum was fixed at $\Gamma=2.3$, as was determined by the pn and NuSTAR spectra, as otherwise this cannot be determined over the soft X-ray band below $2 \mathrm{keV}$, where little of the primary power-law emission is seen in this highly absorbed spectrum.

The mean RGS spectrum, with the best-fit model and components overlaid, is shown in Figure 8. The soft absorber strongly attenuates the absorbed power law (Figure 8, magenta line), which only emerges shortward of $10 \AA$ and is almost fully opaque at longer wavelengths, while the unattenuated powerlaw component (green line) is accounted for in the partial covering model. The parameters of the soft X-ray absorber are similar to what was found for the broadband spectrum: a column of $N_{\mathrm{H}}=6.0_{-2.7}^{+2.3} \times 10^{22} \mathrm{~cm}^{-2}$, an ionization parameter of $\log \xi=3.0 \pm 0.4$, and a line-of-sight covering fraction of $f=0.75_{-0.09}^{+0.06}$.
The broadened XSTAR emission component (cyan line) then accounts for the excess emission between 12 and $17 \AA$ and dominates the spectrum at long wavelengths. Note that this emitter has a large velocity broadening to account for this strong excess, with a Gaussian velocity width of $\sigma_{\mathrm{v}}=$ $30,000_{-6000}^{+12,000} \mathrm{~km} \mathrm{~s}^{-1}$. The column density of the emitter is assumed to be equal to the soft X-ray absorber, where $N_{\mathrm{H}}=6.0 \times 10^{22} \mathrm{~cm}^{-2}$ as above, as this dominates the opacity in this band. Thus, the implicit assumption in the model is that the absorbing gas and emitting gas are essentially the same, with the latter integrated along all sight lines and the former only seen along the direct line of sight. This appears to be supported by the ionization parameter of the emitter, which is $\log \xi=2.74 \pm 0.12$ and is consistent within errors with the ionization of the soft absorber. Note that the broad excess can also be parameterized with a simple Gaussian function, with a width of $\sigma=90_{-40}^{+40} \mathrm{eV}$, at an energy centroid of $837 \pm 17 \mathrm{eV}$. The addition of the emission component significantly improved the fit by $\Delta \chi^{2}=79$, compared to an absorbed power-law continuum. The best-fit parameters of the RGS model are tabulated in Table 4.

A narrower absorption feature also appears to be superimposed on the broadened emission, at a rest-frame wavelength of $\lambda=14.81 \pm 0.09 \AA$ (or $E=837 \pm 5 \mathrm{eV}$ ) and an equivalent width of $-9.5 \pm 4.0 \mathrm{eV}$, and its addition further improved the fit statistic by $\Delta \chi^{2}=23$. If this feature is associated with the strong blueshifted O VIII Ly $\alpha$ doublet at $18.97 \AA$ (or $653.5 \mathrm{eV}$ ), then this would imply an outflow velocity of $v / c=$ $-0.242 \pm 0.009$, which would be consistent with the velocity of $-0.25 c$ obtained from the Fe K absorber (zone 1a). Indeed, this is the strongest soft X-ray absorption line predicted to be present from this zone of gas, as is seen in Figure 6. An identification with a slower (or stationary) absorption line appears less likely; the nearest absorption line at $\lambda_{\text {rest }}=$ $14.82 \AA$ occurs from O VIII $1 s \rightarrow 5 p$, which one would expect to be much weaker compared to the corresponding $1 s \rightarrow 2 p$ line. Thus, an identification with O VIII Ly $\alpha$, at a self-consistent 


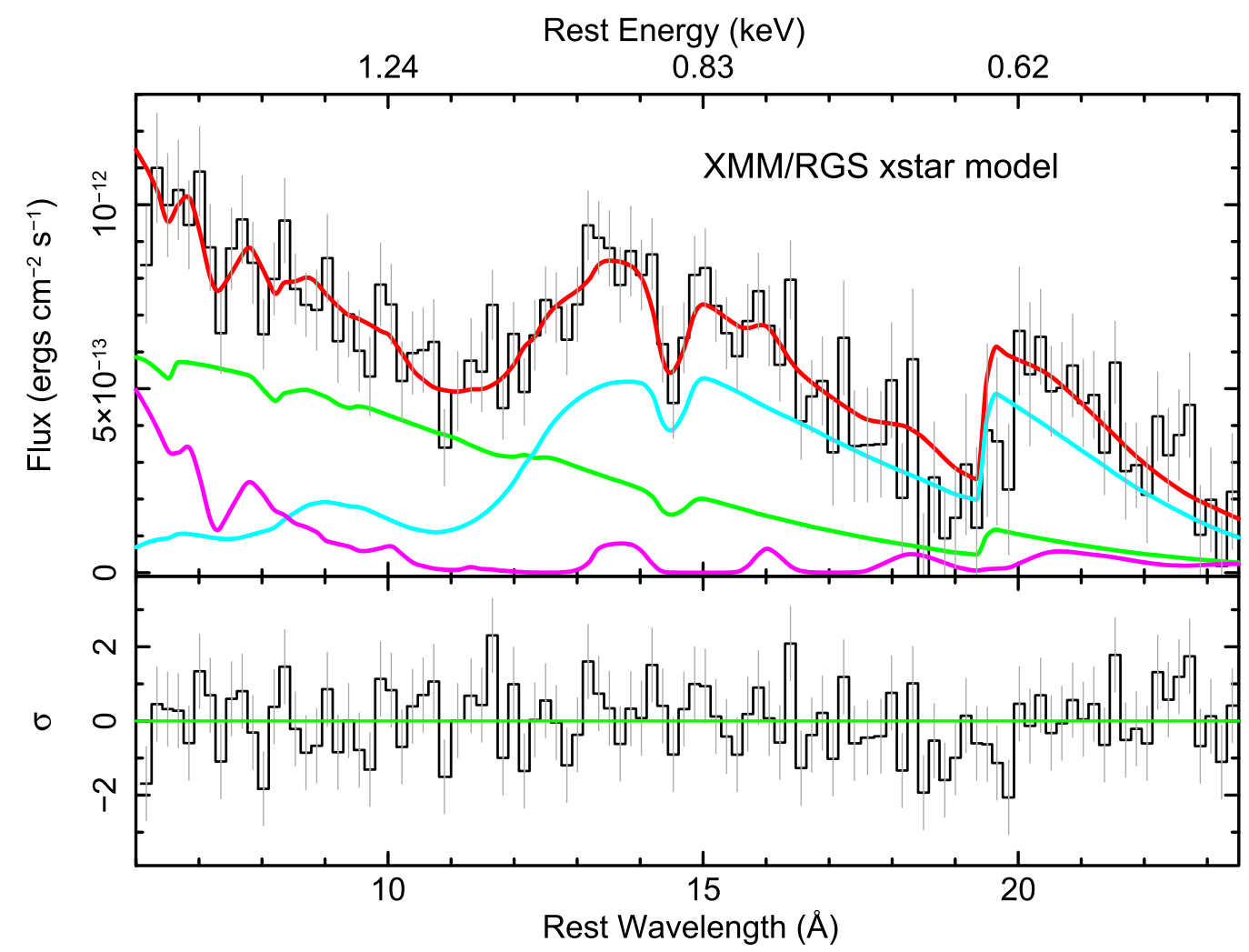

Figure 8. Mean XMM-Newton RGS $1+2$ spectrum of PDS 456, combined for both OBS 1 and OBS 2 to maximize signal-to-noise ratio. Data points are shown as black histograms (with gray error bars), while the best-fit XSTAR model is overlaid in red (solid line). The model components (solid lines) are as per Figure 5. Several features are apparent in the spectrum. A strong, broad excess of emission is observed between 12 and $17 \AA$, which can be modeled by a velocity-broadened emission component from a wide-angle wind (cyan curve). The broad trough between 9 and $12 \AA$ arises from the low-ionization absorption component (zone 2, magneta curve) and is likely produced by a blend of iron L-shell transitions. A significant absorption line is also apparent at $14.5 \AA$. If this is associated with blueshifted O VIII Ly $\alpha$ (at $19.0 \AA$ ), then its outflow velocity of $-0.25 c$ is consistent with the iron $\mathrm{K}$ absorber. Note that the drop near $20 \AA$ ( $23 \AA$ observed frame) arises from the neutral O I edge, associated with absorption from our Galaxy.

velocity as per the $\mathrm{Fe} \mathrm{K}$ absorber, seems the most likely interpretation.

To further test this, we fitted for a high-ionization XSTAR component of the absorber, fully covering the RGS spectrum, in order to account for the possible O VIII Ly $\alpha$ absorption. Indeed, the parameters are consistent with the zone 1a absorber above, with $N_{\mathrm{H}}=2.8_{-1.5}^{+2.0} \times 10^{23} \mathrm{~cm}^{-2}, \log \xi=5.3_{-0.2}^{+0.5}$, and $v / c=-0.260 \pm 0.008$. This suggests that the O VIII absorption originates as part of the same outflow measured at iron $\mathrm{K}$. The final fit statistic, with all these components added (two absorbers plus a soft emitter), is then acceptable, with $\chi_{\nu}^{2}=209 / 218$.

\subsection{The Soft X-Ray Emission}

The flux of the soft X-ray emission can also be used to infer the global covering factor of the outflowing gas; e.g., see Reeves et al. (2018b) for a similar calculation in PG 1211+143. From the photoionization modeling, the normalization (or flux), $\kappa$, of an emission component is defined by XSTAR in terms of

$$
\kappa=f_{\mathrm{cov}} \frac{L_{38}}{D_{\mathrm{kpc}}^{2}},
$$

where $L_{38}$ is the ionizing luminosity over the $1-1000$ ryd band in units of $10^{38} \mathrm{erg} \mathrm{s}^{-1}$ and $D_{\mathrm{kpc}}$ is the distance to the quasar in kpc. Here $f_{\text {cov }}$ is the covering fraction of the gas with respect to the total solid angle, where $f_{\text {cov }}=\Omega / 4 \pi$. For a spherical shell of gas, $f_{\text {cov }}=1$, while $L$ is the quasar luminosity that illuminates the photoionized shell. Thus, by comparing the predicted normalization $(\kappa)$ for a fully covering shell of gas illuminated by a luminosity $L$ versus the observed normalization ( $\kappa_{\text {xstar }}$, as tabulated in Tables 3 and 4$)$ determined from the photoionization modeling, the global covering fraction of the gas can be estimated. For PDS 456, the ionizing luminosity is estimated to be $L=5 \times 10^{46} \mathrm{erg} \mathrm{s}^{-1}$ (Nardini et al. 2015), while its distance is $D=726 \mathrm{Mpc}$. Thus, for a spherical shell of emitting gas the expected XSTAR normalization is $\kappa=9.5 \times 10^{-4}$. As noted above, the global column density of the emitter is assumed to be equal to that of the soft absorber.

Compared to the observed normalization factors reported in Table 4 from the RGS analysis, of $\kappa=(1.03 \pm 0.11) \times 10^{-3}$, the global covering fraction of the soft X-ray-emitting gas is estimated to be $f=1.1 \pm 0.1$, consistent with the gas covering a substantial fraction of $4 \pi$ sr. Note that similar estimates for the global covering fraction are also found for the broadband analysis from the simultaneous pn and NuSTAR spectra (see Table 3). This is consistent with the result obtained by Nardini et al. (2015) for the high-ionization wind measured at Fe K, which was found to cover at least $2 \pi$ sr solid angle. Similar results were also obtained by Reeves et al. (2016) from considering the soft X-ray spectra of the archival XMM-Newton observations of PDS 456.

A more intuitive estimate can be made by comparing the luminosity of the soft X-ray emission component versus the continuum luminosity that is absorbed by the soft X-ray 
Table 4

Parameters for the 2017 RGS Spectrum

\begin{tabular}{lc}
\hline \hline Parameter & Value \\
\hline \multicolumn{3}{c}{ High-ionization Absorber, Zone 1a } \\
\hline$N_{\mathrm{H}}{ }^{\mathrm{a}}$ & $2.8_{-1.5}^{+2.0}$ \\
$\log \xi^{\mathrm{b}}$ & $5.3_{-0.2}^{+0.5}$ \\
$v / c$ & $-0.260 \pm 0.008$ \\
\hline
\end{tabular}

Soft X-Ray Absorber, Zone 2

\begin{tabular}{lc}
\hline$N_{\mathrm{H}}{ }^{\mathrm{a}}$ & $0.60_{-0.27}^{+0.23}$ \\
$\log \xi^{\mathrm{b}}$ & $3.0 \pm 0.4$ \\
$\nu / c$ & $-0.23 \pm 0.02$ \\
$f^{\mathrm{c}}$ & $0.75_{-0.11}^{+0.06}$ \\
\hline
\end{tabular}

\begin{tabular}{lc}
\hline \multicolumn{3}{c}{ Soft X-Ray Emission } \\
\hline$N_{\mathrm{H}}{ }^{\mathrm{a}}$ & $0.60^{\mathrm{d}}$ \\
$\log \xi^{\mathrm{b}}$ & $2.74_{-0.10}^{+0.12}$ \\
$\kappa_{\mathrm{xstar}}^{\mathrm{e}}$ & $(1.03 \pm 0.11) \times 10^{-3}$ \\
$\Omega / 4 \pi^{\mathrm{f}}$ & $1.1 \pm 0.1$ \\
\hline
\end{tabular}

\begin{tabular}{lc}
\hline & Power-law Continuum \\
\hline$\Gamma$ & $2.3^{\mathrm{d}}$ \\
$N_{\mathrm{ABS}}{ }^{\mathrm{g}}$ & $0.55 \pm 0.07$ \\
$N_{\mathrm{UNABS}}{ }^{\mathrm{g}}$ & $1.6 \pm 0.5$ \\
$F_{0.5-2.0 \mathrm{keV}^{\mathrm{h}}}$ & 1.02 \\
\hline
\end{tabular}

Notes.

${ }^{\mathrm{a}}$ Units of column density $\times 10^{23} \mathrm{~cm}^{-2}$.

${ }^{\mathrm{b}}$ Ionization parameter in units of $\mathrm{erg} \mathrm{cm} \mathrm{s}^{-1}$.

${ }^{c}$ Covering fraction of absorber.

${ }^{\mathrm{d}}$ Denotes that the parameter is fixed.

e Measured normalization of XSTAR emission component, as described in Table 3.

${ }^{\mathrm{f}}$ Solid angle of the emission component, as per Table 3.

${ }^{\mathrm{g}}$ Relative normalizations of the absorbed and unabsorbed power-law continuum components. Normalizations are in flux units of $\times 10^{-3}$ photons $\mathrm{cm}^{-2} \mathrm{~s}^{-1} \mathrm{keV}^{-1}$ at $1 \mathrm{keV}$.

${ }^{\text {h }}$ Observed 0.5-2 keV flux, not corrected for absorption, in units of $\times 10^{-12} \mathrm{erg} \mathrm{cm}^{-2} \mathrm{~s}^{-1}$.

absorber. If the gas subtends a solid angle close to $4 \pi$ sr and the continuum emission is isotropic, the above ratio will be close to 1 , as most of the radiation that is absorbed will be subsequently re-emitted. Using the above spectral fit for the broadband NUSTAR and pn data, the X-ray luminosity of the emitter is $L_{0.3-10 \mathrm{keV}}=1.5 \times 10^{44} \mathrm{erg} \mathrm{s}^{-1}$, while the luminosity absorbed is $L_{0.3-10 \mathrm{keV}}=4.7 \times 10^{44} \mathrm{erg} \mathrm{s}^{-1}$, and thus the above ratio is $\sim 0.3$. Thus, approximately $30 \%$ of the absorbed continuum emission is subsequently re-emitted in the soft $\mathrm{X}$-ray band, which is consistent with a relatively high global covering fraction of the gas. The soft X-ray emission component dominates in these low-flux observations owing to the intrinsic continuum being highly absorbed, which revealed the reprocessed components originating from the wind.

As noted above, the velocity broadening of the emission was found to be very high, with $\sigma_{\mathrm{v}}=30,000 \mathrm{~km} \mathrm{~s}^{-1}$, as can be seen from the broad emission present below $1 \mathrm{keV}$. Note that, by itself, no net velocity shift is required for the emitter. However, the velocity width is consistent with the observer viewing both the red- and blueshifted emission integrated across all lines of sight toward a wide-angle expanding wind. Indeed, the FWHM velocity of the emitter, which would arise across all sight lines, is similar in magnitude to the net outflow velocity of $0.25 c$ that is viewed directly along the line of sight. This may suggest that the soft X-ray emission and absorption arise from the same wide-angle wind.

\section{Multiwavelength Observations of PDS 456}

\subsection{Simultaneous HST Observation}

Simultaneously with the XMM-Newton/NuSTAR observations, a UV spectrum of PDS 456 was also acquired with the Cosmic Origins Spectrograph (COS) on board HST, using the G140L grating. Note that this was the first simultaneous UV versus X-ray spectrum of PDS 456, where the earlier archival observations taken with HST/STIS in 2000 (O'Brien et al. 2005) and with HST/COS in 2014 (Hamann et al. 2018) were not concurrent with any of the past X-ray observations. The data were retrieved from the Mikulski Archive for Space Telescopes with no further reprocessing. The $H S T$ exposure fell astride the beginning of the first XMMNewton observation (OBS 1), for a total net time of $4643 \mathrm{~s}$. Following Hamann et al. (2018), we corrected for Galactic reddening by adopting the extinction law from Cardelli et al. (1989) with $R_{V}=3.1$ and $E(B-V)=0.45$. The continuum in 2017 was then fitted as a power law over line-free wavelength intervals, returning an index of $\alpha_{\lambda}=-1.70$, virtually coincident with the value estimated for the 2000 observation $(-1.68)$. Indeed, from a simple visual inspection, the 2000, 2014, and 2017 HST spectra of PDS 456 have a remarkably similar continuum shape, once the different flux levels are taken into account. There are some modest variations in the continuum flux, where the earliest 2000 spectrum shows the highest flux recorded so far, which had dropped by about $1 / 3$ by 2014 before slightly recovering to about $4 / 5$ of the original flux in 2017.

The 2017 HST/COS versus 2000 HST/STIS spectra are shown in Figure 9, where the dereddened spectra have been plotted as a ratio against the best-fit continuum slope described above. The broad emission line profiles from Ly $\alpha$ (also blended with $\mathrm{NV}$ ), C IV, and Si IV are consistent between epochs, with the lines having a marginally higher equivalent width in the 2017 epoch, compared to 2000 . Note that the emission-line profiles from the 2000 and 2014 epochs are discussed in detail in O'Brien et al. (2005) and Hamann et al. (2018), where in particular the CIV emission profile is blueshifted by $-5000 \mathrm{~km} \mathrm{~s}^{-1}$.

In the context of this paper, it is worth checking the spectra for the presence of any BAL-like features, in order to gain further insights on the physical relation between the (soft) X-ray and UV absorbers. The latter has manifested itself in the form of a broad absorption trough at $1346 \AA$ (observed) in the HST/STIS spectrum taken in 2000 , first revealed by O'Brien et al. (2005) and recently identified as a possible C IV line outflowing at $\sim 0.3 c$ by Hamann et al. (2018). In agreement with the $2014 \mathrm{HST} / \mathrm{COS}$ spectrum, there is no evidence for a prominent trough in the 2017 data shortward of the Ly $\alpha$ emission line, which only appears to be present in the 2000 spectrum, but just a hint of a shallow depression. Figure 9 shows a comparison over the band of interest between the 2000 and 2017 spectra, normalized to the continuum level and visually rebinned for clarity. We included a Gaussian profile to reproduce any possible absorption feature at the position of the 


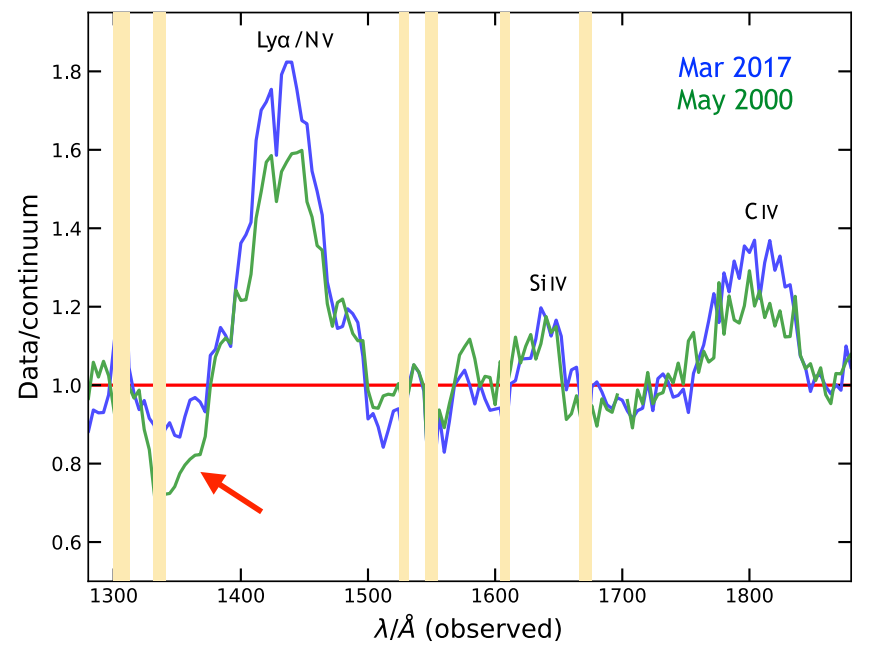

Figure 9. Comparison between the 2000 and 2017 UV epochs of PDS 456 observed with $H S T$. Note that the narrow yellow bands mark Galactic features. In the $2000 \mathrm{HST} / \mathrm{STIS}$ spectrum, a broad absorption trough (red arrow) is observed at $\sim 1350 \AA$ ( $1140 \AA$ rest frame), blueward of Ly $\alpha$. The BAL feature may be identified with Ly $\alpha$ or $\mathrm{N} V$ shifted by $0.06 c-0.08 c$, or with a C IV BAL at $\sim 0.3 c$ (Hamann et al. 2018). The BAL feature has substantially weakened in 2017 March, despite the X-rays being highly absorbed.

putative C IV blueshifted line. We obtained a central (observed) wavelength $\lambda_{\mathrm{o}}=1339.1 \pm 1.5 \AA$ (against 1346.1 $\pm 0.6 \AA$ for the 2000 feature; see Hamann et al. 2018), optical depth $\tau_{\mathrm{o}}=$ $0.14 \pm 0.01$ (against $0.35 \pm 0.01$ ), and a Doppler broadening of $b=6321 \pm 718 \mathrm{~km} \mathrm{~s}^{-1}$ (against $5135 \pm 203 \mathrm{~km} \mathrm{~s}^{-1}$ ). We stress, however, that in the 2017 spectrum the properties of the very shallow trough heavily depend on the exact level (and slope) of the continuum. Thus, we do not consider the detection of this feature as fully reliable compared to the deeper 2000 trough, and we take its optical depth as an indicative upper limit.

\subsection{The Optical to X-Ray Spectral Energy Distribution}

We constructed the SED of PDS 456 during the 2017 observations, also using the data from OBS 1 during the minimum in X-ray flux. It may be the case that the lower ionizing flux during the current observations affects the overall properties of the absorber, and thus it is instructive to compare the 2017 SED with that obtained during previous observations. The 2017 SED was constructed using the same phenomenological form as in Nardini et al. (2015) and Matzeu et al. (2016), where a double broken power-law form was used for the continuum from the optical to hard X-ray band. Simultaneous photometry from the XMM-Newton OM was used, in the $V, B, U$, UVW1, UVW2, and UVM2 bands, as well as simultaneous photometry taken with the $V$ and UVW1 filters from the Swift UVOT, which was also coincident with OBS 1. The optical/UV data points were corrected for a reddening as per the HST observations. Both the soft X-ray and the iron $\mathrm{K}$ absorption components are included in the model, as parameterized above in Table 3 , as well as the Galactic absorption. The underlying continuum shape can then be described by three photon indices: $\Gamma=1.1 \pm 0.1$ in the optical/UV band up to a break energy of $10 \mathrm{eV}$, a steep photon index of $\Gamma=3.33 \pm 0.05$ connecting the UV to the soft X-ray band up to a break energy of $500 \mathrm{eV}$, and a photon index of $\Gamma=2.34 \pm 0.06$ from 0.5 to $40 \mathrm{keV}$ in the X-rays.
The 2017 SED is shown in Figure 10, where the emission peaks in the UV band compared to the X-ray. Overall the $1-1000$ ryd ionizing luminosity of this SED is $L=3.5 \times$ $10^{46} \mathrm{erg} \mathrm{s}^{-1}$, corrected for Galactic absorption and reddening, but not for any intrinsic absorption from the wind. The effect of the X-ray absorption is apparent in the figure, where the dotted blue line shows the intrinsic continuum level and the solid line the continuum after absorption through the wind. The primary attenuation occurs in the soft X-ray band, where a maximum in absorption opacity occurs near $1 \mathrm{keV}$. However, the bolometric output is dominated by the optical/UV band, which, as discussed above, appears relatively unaffected by the presence of the X-ray absorber.

The 2017 SED was also compared with what was computed by Nardini et al. (2015), on the basis of the 2013-2014 XMMNewton and NUSTAR observations. We reconstructed the SED from the first sequence of that campaign (OBS A), which was also the brightest and least absorbed of those five earlier sequences; the SED model of the 2013 observation is shown in Figure 10 (green dot-dashed line). The 1-1000 ryd luminosity is only slightly higher in 2013 OBS A, with $L=5.0 \times$ $10^{46} \mathrm{erg} \mathrm{s}^{-1}$. The main difference between 2013 OBS A and the 2017 SEDs lies in the X-ray band, where the observed $0.3-30 \mathrm{keV}$ band luminosity has decreased by a factor of $\times 4$, from $L_{\mathrm{X}}=2.0 \times 10^{45} \mathrm{erg} \mathrm{s}^{-1}$ to $L_{\mathrm{X}}=5.7 \times 10^{44} \mathrm{erg} \mathrm{s}^{-1}$. Thus, while the X-ray luminosity has decreased between these observations, the optical/UV portion remained unchanged. Indeed, as will be discussed in a subsequent paper on the longterm X-ray versus UV variability from the 2017 Swift campaign, most of the pronounced variability occurs in the X-ray band, with relatively little $(<10 \%)$ change in the UV flux over a period of 6 months. We note that a similar behavior can occur in other AGNs, for instance, in the Seyfert 1 Mrk 335, where large-amplitude X-ray variability is present, but the UV variability is less pronounced (Gallo et al. 2018).

\section{Discussion}

\subsection{Properties of the Soft X-Ray Absorber}

We now discuss the possible location and properties of the soft X-ray wind in PDS 456, compared to the well-studied outflow that is present at iron $\mathrm{K}$. The previous campaigns on PDS 456 were able to place constraints on the location of the iron K outflow, based on its variability. Nardini et al. (2015) estimated a likely radius of $\sim 200 R_{\mathrm{g}}{ }^{9}$ for the $0.25 \mathrm{c} \mathrm{Fe} \mathrm{K}$ absorber. This was based on the typical response time, of 1 week, of the P Cygni like profile at Fe $\mathrm{K}$ to changes in the hard $\mathrm{X}$-ray continuum, as measured during the series of five simultaneous XMM-Newton and NuSTAR observations in 2013-2014. Rapid variability of the iron K absorber was also observed during the 2013 Suzaku observations of PDS 456, which covered a baseline of $\sim 1$ Ms (Gofford et al. 2014; Matzeu et al. 2016). PDS 456 had a low X-ray flux during those observations, and the iron $\mathrm{K}$ absorber increased by a factor of $\times 10$ in equivalent width during the Suzaku campaign. Based on this variability, Matzeu et al. (2016) estimated a distance of $R \sim 200 R_{\mathrm{g}}=3 \times 10^{16} \mathrm{~cm}$, assuming that the variability was due to an increase in the column density of the outflow. A slightly larger radial distance estimate was obtained by Gofford et al. (2014), of about $R \sim 1000 R_{\mathrm{g}} \sim 10^{17} \mathrm{~cm}$, from the

\footnotetext{
9 Note that in PDS 456, with a likely black hole mass of $10^{9} M_{\odot}$ (Reeves et al. 2014), $1 R_{\mathrm{g}}=G M / c^{2}=1.5 \times 10^{14} \mathrm{~cm}$.
} 


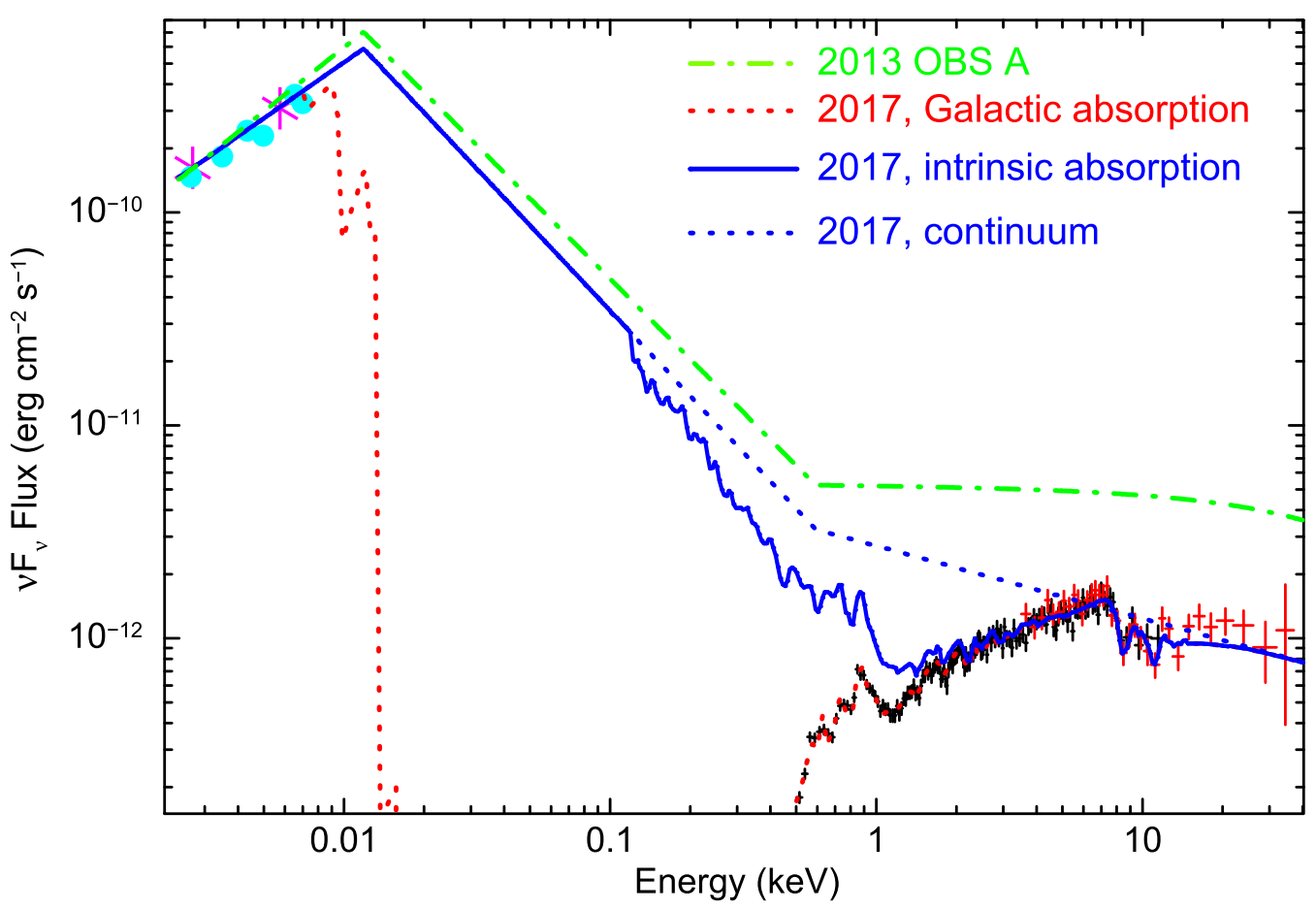

Figure 10. The 2017 optical/UV to X-ray SED of PDS 456. Data points are taken from the OBS 1 sequence, consisting of XMM-Newton pn (black crosses), NuSTAR (red crosses), Swift UVOT (magenta crosses), and XMM-Newton OM (cyan circles). The optical/UV photometric points are corrected for reddening. The figure shows various models fitted to the SED, using a double broken power-law continuum. The red dotted line shows the SED model, absorbed by the Galactic column, as well as by intrinsic absorption from the wind. The blue solid line shows the model corrected for Galactic absorption, but still attenuated by the wind, while the dashed blue line shows the intrinsic continuum after all absorption is removed. Note that the wind mainly attenuates the soft X-ray band. In contrast, the green dot-dashed line shows the intrinsic continuum from the brighter, 2013 OBS A XMM-Newton and NuSTAR observation, which was largely unabsorbed by the wind; see Nardini et al. (2015) for details. The 2017 observation is a factor of $\times 4$ fainter in the X-ray band compared to 2013, although there is little difference in the optical/UV.

recombination timescale, on the assumption that the ionization of the iron $\mathrm{K}$ absorber responded to the decrease in the X-ray continuum flux during those observations.

An interesting question is, what is the location of the lowerionization soft X-ray absorber, relative to the iron $\mathrm{K}$ absorber? Adopting the above distance of $R=3 \times 10^{16} \mathrm{~cm}$ for the iron $\mathrm{K}$ wind, then for an ionization of $\log \xi=5$ (Table 3, zone 1a) and an ionizing luminosity of $3 \times 10^{46} \mathrm{erg} \mathrm{s}^{-1}$ (Section 2), its density is $n_{\mathrm{e}}=L / \xi R^{2} \sim 3 \times 10^{8} \mathrm{~cm}^{-3}$. In contrast, the soft $\mathrm{X}$-ray absorber is $\times 100$ less ionized, with $\log \xi=3$, and so this would require its density to be $\times 100$ higher to compensate, i.e., $n_{\mathrm{e}} \sim 3 \times 10^{10} \mathrm{~cm}^{-3}$. Given the soft X-ray column density of $N_{\mathrm{H}}=6 \times 10^{22} \mathrm{~cm}^{-2}$, then the absorber size scale is $\Delta R \sim N_{\mathrm{H}} / n_{\mathrm{e}}=2 \times 10^{12} \mathrm{~cm}$. This seems less likely, as it implies that the soft X-ray absorber exists in the form of many dense but extremely small clumps, of $\ll 1 R_{\mathrm{g}}$ in size, so as to not become overionized close to the X-ray source.

Instead, the soft X-ray absorber could be located farther out than the high-ionization zones of the wind, where a factor of $\times 10$ increase in distance would translate to a decrease in ionization of $\times 100$ for a given density. We can place an estimate on the radial location of the soft X-ray absorber in PDS 456 from its variability between OBS 1 and OBS 2. In particular, during the dip in OBS 1, the soft X-ray absorber appeared to reach a maximum covering of $100 \%$ of the X-ray source, which then decreased to about 70\% during OBS 2 as the AGN brightened. Thus, we likely witnessed an occultation event, whereby the absorbing clump or cloud fully covered the $\mathrm{X}$-ray source during the dip and then gradually moved out of the line of sight as the X-ray observations progressed.
One possible assumption is that the absorber size scale $(\Delta R)$ is approximately equal to the X-ray source size $(D)$. If the absorbing cloud were much smaller, then it would not be able to cover such a high fraction (up to $100 \%$ ) of the X-ray source, while if it were substantially larger, the variations in covering fraction would likely not be seen. The X-ray source or coronal size can be estimated from the light-crossing time, i.e., $D=c \Delta t$, where $\Delta t$ corresponds to the doubling time of the $\mathrm{X}$-ray flares. Figures 1 and 2 show various rapid $\mathrm{X}$-ray flares that double in flux on timescales of tens of kiloseconds, for instance, at $280 \mathrm{ks}$ into the NUSTAR observation or after 3 days into the Swift monitoring. Indeed, such rapid X-ray flaring has been noted previously in PDS 456, from RXTE, Beppo-SAX, and Suzaku observations (Reeves et al. 2000, 2002; Matzeu et al. 2016), and implies that the X-ray source is a few gravitational radii in extent.

If the obscuring cloud has a size scale $\Delta R \approx D=c \Delta t$, with column density $N_{\mathrm{H}}=n_{\mathrm{H}} \Delta R$ (where the electron density $\left.n_{\mathrm{e}} \sim n_{\mathrm{H}}\right)$ and ionization parameter $\xi=L_{\mathrm{ion}} / n_{\mathrm{e}} R^{2}$, then its distance, $R$, is

$$
R \approx\left(\frac{L_{\mathrm{ion}} c \Delta t}{N_{\mathrm{H}} \xi}\right)^{1 / 2}
$$

From the X-ray spectral analysis, the column density of the soft X-ray absorber is $N_{\mathrm{H}}=6 \times 10^{22} \mathrm{~cm}^{-2}$, while its ionization is $\log \xi=3$ (see Table 3). The ionizing luminosity, as defined by XSTAR over the $1-1000$ ryd range, is $L=3 \times$ $10^{46} \mathrm{erg} \mathrm{s}^{-1}$, measured from the SED analysis in Section 2.

The doubling time is estimated to be $\Delta t=20 \mathrm{ks}$ from the X-ray flares, and thus $D=6 \times 10^{14} \mathrm{~cm}$ (or $\sim 10^{15} \mathrm{~cm}$ ). The 
above values yield $R=5 \times 10^{17} \mathrm{~cm}$ or $\sim 0.1 \mathrm{pc}$. This is consistent with a similar estimate in Reeves et al. (2016), based on an analysis of the soft X-ray absorber variations over the previous XMM-Newton observations of PDS 456 from 2001 to 2014. The larger radial distance to the soft X-ray gas, compared to the high-ionization absorber, likely prevents it from becoming overionized. At this distance the Keplerian velocity is $\sim 5000 \mathrm{~km} \mathrm{~s}^{-1}$, while the gas density is $n_{\mathrm{e}} \sim 10^{8} \mathrm{~cm}^{-3}$, consistent with a BLR scale origin.

The transverse velocity of the absorbing clouds can also be estimated, using the time taken for the X-ray source to uncover. In Section 3.3, we determined that the absorber covering fraction varied from $f>0.97$ during the dip in OBS 1 to $f=0.83 \pm 0.02$ in the remainder of OBS 1 , and then declining further to $f=0.73 \pm 0.03$ in OBS 2. Thus, the change in covering fraction is $\Delta f \sim 0.25$, over a baseline of $\Delta t_{\text {uncov }}=$ $150 \mathrm{ks}$ (i.e., one XMM-Newton orbit). The cloud transverse velocity is then

$$
v_{\mathrm{t}} \approx \frac{\Delta f D}{\Delta t_{\mathrm{uncov}}} .
$$

This gives a transverse velocity of $v_{\mathrm{t}} \sim 10^{4} \mathrm{~km} \mathrm{~s}^{-1}$. This is within a factor of two of the Keplerian velocity, while the wind may also have a transverse component across the line of sight. These transiting wind clumps may provide an explanation for the variable partial covering previously seen in the 2013 Suzaku observations of PDS 456 (Matzeu et al. 2016), where the QSO was in a similar low-flux state and substantial spectral variability was also observed.

A similar scenario was also recently suggested for the fast outflow in the quasar PG $1211+143$ (Reeves et al. 2018b). Here, the fast wind consists of a two-phase (low- and highionization) clumpy medium, where the smaller, denser lowionization clumps may account for the rapid variations in the soft X-ray absorbing gas on timescales of days. Such a scenario may also be required in BAL quasars, in order to explain the coexistence of both the UV and X-ray absorbing gas (Hamann et al. 2013). Recently, a rapid $(\Delta t=100 \mathrm{ks})$ and large $\left(\Delta N_{\mathrm{H}}=10^{24} \mathrm{~cm}^{-2}\right)$ variation in the absorbing column was measured in the fast $(\sim 0.1 c)$ wind in the Seyfert 2, MCG-3-58007, during an X-ray occultation event (Braito et al. 2018). This was likely due to a denser wind streamline moving across the line of sight, which occurred during the course of a NUSTAR observation. Indeed, similar to the case of PDS 456, the decline in flux was measured only in the softer X-ray bands below $10 \mathrm{keV}$, whereas the hard X-ray continuum did not vary during the absorption event.

Overall, it appears likely that the accretion disk winds are not homogeneous in nature, and the passage of dense, high column density clumps could explain their short-timescale variability, as is predicted in time-dependent wind simulations (Proga et al. 2000; Proga \& Kallman 2004). Furthermore, the X-ray eclipse in PDS 456 may be related to X-ray obscuration events that occur in other AGNs (Markowitz et al. 2014), but at lower outflow velocities. For example, the Seyfert 1 NGC 3783 has recently dropped into a low X-ray flux state, owing to a pronounced increase in X-ray absorption (Mehdipour et al. 2017), and this may be caused by frequent obscuration events (Kaastra et al. 2018). Notably, a long-term (and ongoing) increase in obscuration has been seen toward NGC 5548 (Kaastra et al. 2014). A rapid X-ray absorption event has also been recently detected in XMM-Newton observations of the
Seyfert 1 galaxy NGC 3227 (Turner et al. 2018), as was evident through a similar pronounced dip in the soft X-ray light curve, which was accompanied by an increase in the soft X-ray column or covering fraction. The opposite can also occur, in the form of uncovering events, as was found, for example, in the classic changing-look AGN NGC 1365 (Braito et al. 2014). In all of these cases, the rapidly variable X-ray obscuration is likely linked with inhomogeneous matter, possibly originating from an accretion disk wind. In PDS 456, the event is only more extreme owing to the large velocity of the outflowing gas.

\subsection{The Ultrafast Zone}

In contrast to the soft X-ray absorber, the fastest $(\sim 0.4 c)$, highest-ionization $(\log \xi=6)$ phase of the wind likely originates from closest to the black hole, while the column of this component may be as high as $N_{\mathrm{H}}=7 \times 10^{23} \mathrm{~cm}^{-2}$ (OBS 1, Table 3, zone 1b). An upper limit to its radial distance arises from the geometric condition that $\Delta R / R<1$, which, when combined with the electron density of $n_{\mathrm{e}}=N_{\mathrm{H}} / \Delta R$ and the definition for the ionization parameter, yields $R<L / N_{\mathrm{H}} \xi$; see also Tombesi et al. (2013) or Gofford et al. (2015) for similar radial estimates. For the above parameters, $R<4 \times 10^{16} \mathrm{~cm}$, which is consistent with the estimate of $\sim 200 R_{\mathrm{g}}$ in Nardini et al. (2015) for the $0.25 c$ iron $\mathrm{K}$ absorber. A minimum distance derives from the escape radius, where $R_{\mathrm{esc}}=2\left(c^{2} / v^{2}\right) R_{\mathrm{g}}=$ $10 R_{\mathrm{g}} \sim 10^{15} \mathrm{~cm}$.

While the exact distance of the fastest zone is not well determined, the above estimates are consistent with it being an innermost streamline of the wind, which, given its velocity, may be launched from close to the black hole. As was discussed in Paper I, this fast phase might only be detected when the incident X-ray flux is relatively low, as per these 2017 observations, so that the gas is not fully ionized. The only previous observations of PDS 456 where the X-ray flux was this low occurred during the long 2013 Suzaku campaign. However, those observations lacked the sensitivity of NuSTAR above $10 \mathrm{keV}$ for detecting this putative high-velocity wind component. Further NUSTAR observations will test whether the ultrafast zone is only apparent during observations at low $\mathrm{X}$-ray flux.

\subsection{UV versus $X$-Ray Absorption}

In the original $2000 \mathrm{HST} / \mathrm{STIS}$ spectrum of PDS 456 (O'Brien et al. 2005), a broad absorption trough was observed at $1346 \AA$ (1140 $\AA$ rest frame), blueshifted by $-0.06 c$ with respect to the strong broad Ly $\alpha$ emission line (see Figure 9). Hamann et al. (2018) recently reanalyzed those data and found that this trough is unlikely to be associated with Ly $\alpha$ owing to the very low ionization that is required, without other lines from metal ions being present. They suggested that the trough is most likely either associated with $\mathrm{N} V \lambda 1240$, blueshifted by $-0.08 c$, or from highly blueshifted C IV $\lambda 1549$ at a velocity of $-0.3 c$. Note that the latter velocity is also commensurate with the outflow velocity typically measured in the X-rays from the Fe K profile (Reeves et al. 2009; Matzeu et al. 2017). Despite the substantial X-ray absorption seen toward PDS 456 in the simultaneous 2017 observations, the feature at $1346 \AA$ appears to have decreased in depth between 2000 and 2017, where the optical depth of the trough declined from $\tau=0.35$ to $\tau=0.14$ (see Section 4.1). Unlike the X-ray observations, the simultaneous 2017 HST/COS observation of PDS 456 shows 


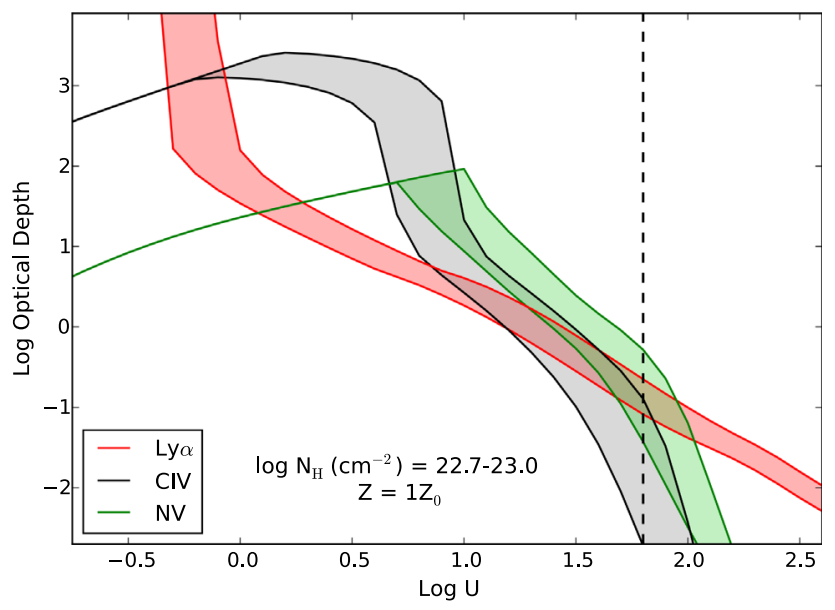

Figure 11. CLOUDY simulations of the UV absorption trough seen in PDS 456. The simulations predict the optical depth of the trough at $1346 \AA$, assuming a BAL origin associated with either Ly $\alpha$ (red), C IV (black), or N V (green), as described in the text. The depth is computed for each ion as a function of the ionization $(\log U)$ and for solar metallicity, where the shaded regions correspond to column densities in the range of $\log \left(N_{\mathrm{H}} / \mathrm{cm}^{-2}\right)=22.7-23.0$, as per the observed soft X-ray column. The vertical dotted line shows an ionization of $\log U=1.8$, corresponding to the soft X-ray absorber and where the UV absorption trough is predicted to be shallow, where typically $\tau \sim 0.1$. Note that the optical depths are a very sensitive function of the ionization; thus, at much lower ionizations than observed here considerably deeper BAL troughs are predicted.

little intrinsic absorption in the UV, while most of the absorption toward PDS 456 occurs in the soft X-ray band.

The highly ionized component of the wind, with $\log \xi=5$, is far too highly ionized to produce detectable absorption feature in the UV, as most of the absorption is associated with $\mathrm{He}$ and H-like iron and only trace amounts of soft X-ray absorption are present, such as from O VIII Ly $\alpha$. However, the lower-ionization soft X-ray absorber, with $\log \xi=3$, may produce detectable UV absorption from the wind. To test this, we ran CLOUDY (Ferland et al. 1998) simulations for a single ionized cloud, using the same methods described in Hamann et al. (2018), over a range of possible ionizations and for soft X-ray column densities of $\log \left(N_{\mathrm{H}} / \mathrm{cm}^{-2}\right)=22.7-23.0$, consistent with what is observed in the 2017 X-ray observations. The simulations assume a turbulence velocity of $b=5135 \mathrm{~km} \mathrm{~s}^{-1}$, which was inferred by Hamann et al. (2018) from fitting the UV absorption trough in 2000, while solar metallicity is assumed. Note that, for the SED of PDS 456, the predicted conversion between $\log U$ and $\log \xi$ is approximately $\log \xi=\log U+1.2$. Thus, an ionization within CLOUDY of $\log U=1.8$ is equivalent to the ionization of $\log \xi=3$ inferred by XSTAR for the soft X-ray absorber.

Figure 11 shows the predicted optical depth for various lines, such as $\mathrm{CIV}, \mathrm{N}$, or $\mathrm{Ly} \alpha$, versus ionization parameter $\log U$. Note that the shaded area of each curve for a particular ion is bounded by the lower $\left(\log \left(N_{\mathrm{H}} / \mathrm{cm}^{-2}\right)=22.7\right)$ and upper $\left(\log \left(N_{\mathrm{H}} / \mathrm{cm}^{-2}\right)=23.0\right)$ values of the column density, to highlight the range of uncertainty. Thus, for the ionization $(\log U=1.8)$ and column expected from the soft X-ray absorber, only small optical depths are predicted for the BAL absorption troughs. For CIV, the predicted optical depth is $\tau<0.1$ (and similar for $\mathrm{NV}$ ), which is consistent with the 2017 HST observation where even if any BAL troughs are present, they are very weak and shallow. Thus, the soft X-ray absorber observed in 2017 is likely to be too ionized to produce substantial UV absorption, and only trace amounts of these ions are present at these ionizations.

However, as can be seen in Figure 11, the optical depths inferred from the CLOUDY model are very sensitive to the ionization. Indeed, the predicted optical depths can increase by more than an order of magnitude, to $\tau>1$, for a decrease in ionization of $\Delta(\log U)=-0.5$. Furthermore, the optical depth is also sensitive to the total hydrogen column density. If the column density exceeds $N_{\mathrm{H}}>10^{23} \mathrm{~cm}^{-2}$, then the ionic fractions of both $\mathrm{C} I \mathrm{~V}$ and $\mathrm{N} \mathrm{V}$ can increase rapidly, owing to the effects of shielding within an individual cloud as it becomes optically thick (see, e.g., Figure 8 of Hamann et al. 2018). Thus, only a modest change in column or ionization could explain the appearance (or disappearance) of the UV trough. Indeed, the soft X-ray column densities toward PDS 456 in the highly obscured 2013 Suzaku observations (Matzeu et al. 2016) were even higher than observed here, exceeding $N_{\mathrm{H}}>$ $10^{23} \mathrm{~cm}^{-2}$. Unfortunately, no simultaneous UV observations were obtained during the 2013 Suzaku epoch, while no simultaneous X-ray observations were available from the 2000 HST epoch when the deep UV trough was apparent.

It is also possible that the UV observations just missed the X-ray obscuration event in the 2017 observations. The HST observation coincided with the start of the first XMM-Newton observation, just prior to the onset of the dip in light curve in Figure 1 and thus the subsequent increase in soft X-ray obscuration. The line of sight toward the UV and X-ray emission regions may also be different. The X-ray emission region is likely located closer to the black hole, and thus an outflowing absorbing cloud, which initially passes in front of the central X-ray source, may be delayed in passing across the UV emitter. The UV emitter may also be more extended than the compact X-ray corona, and therefore the covering fraction of the absorbing clouds could be much smaller against the UV continuum, which as a result would imprint only very shallow UV absorption troughs. Indeed, as was inferred earlier in Section 5.1 from the short timescale of the X-ray eclipse event, the absorbing cloud responsible for the increase in X-ray column density toward PDS 456 is likely to be compact, around 10 gravitational radii in extent. Such a small absorbing cloud would have a lower covering fraction compared to the extended optical/UV emission region, where the latter may originate on scales of tens to hundreds of gravitational radii on the accretion disk. Nonetheless, two further epochs of simultaneous UV and X-ray observations of PDS 456 have been scheduled with $H S T$, $X M M-N e w t o n$, and NuSTAR over the next year, which will test whether there is any link between the UV and soft X-ray absorbers.

\subsection{The Link between UV BALs and X-Ray Ultrafast Outflows}

In general, there appear to be very few examples of ultrafast outflows, discovered in the X-ray band, where there is a corresponding UV counterpart. The nearby $(z=0.0809)$ nonBAL QSO PG 1211+143, which has a well-established X-ray ultrafast outflow (Pounds et al. 2003; Pounds \& Reeves 2009; Reeves et al. 2018b), has recently been found to have a broad UV absorption trough, blueshifted with respect to Ly $\alpha$ by $-17,000 \mathrm{~km} \mathrm{~s}^{-1}$ (Kriss et al. 2018b). This is consistent with the blueshift (of $-0.06 c$ ) of the soft X-ray absorber, as measured in contemporary X-ray grating observations (Danehkar et al. 2018; Reeves et al. 2018b) with XMM-Newton RGS and 
Chandra HETG, as well as with the velocity at iron K (Pounds et al. 2016). In PG 1211+143, the UV mini-BAL trough also appears to be variable and is not detected in all of the epochs analyzed by Kriss et al. (2018b). Thus, aside from PDS 456 and PG 1211+143, as of yet there are no other known examples of UV systems that may be associated with X-ray-selected ultrafast outflows. Indeed, Kriss et al. (2018a) perform a follow-on study of 16 archival UV HST and FUSE observations of ultrafast outflows selected in the X-ray band and do not confirm any detections of blueshifted UV absorption troughs. They suggest that most of the X-ray-selected ultrafast outflows are likely to be too highly ionized to have detectable UV absorption features.

However, the reverse is not the case, and there are several examples of known UV BAL or mini-BAL systems where ultrafast outflows have subsequently been detected in the X-ray band. The most-studied example is the high-redshift $(z=3.9)$ BAL QSO APM 08279+5255, where several observations have clearly established the presence of an ultrafast outflow at iron $\mathrm{K}$ with a typical velocity of $0.2 c-0.4 c$ (Chartas et al. 2002, 2009; Saez et al. 2009; Saez \& Chartas 2011), while, similar to PDS 456, pronounced outflow variability has also been measured. Other recent examples include the BAL QSOs HS 08104+2554 (Chartas et al. 2014) and H 1413+117 (Chartas et al. 2007), as well as the mini-BAL PG $1115+080$ (Chartas et al. 2003). These QSOs are all at high redshift $(z=1-3)$, are gravitationally lensed (boosting their X-ray flux), and have outflow velocities measured at $\mathrm{Fe} \mathrm{K}$ of between $0.1 c$ and $0.4 c$.

While the majority of UV BAL systems are detected at high redshift, as their rest-frame UV spectra then fall into the optical band, there are also notable examples of UV BALs/mini-BAL systems at low redshift that also have corresponding X-ray ultrafast outflows. Mrk 231 is a well-known nearby $(z=0.042)$ low-ionization BAL (LoBAL) QSO, which is also classified as an ultraluminous infrared galaxy (Sanders et al. 2003). Feruglio et al. (2015) measured both the presence of a fast X-ray wind, with an outflow velocity of $-20,000 \mathrm{~km} \mathrm{~s}^{-1}$, and a large-scale molecular outflow in $\mathrm{CO}$, the latter with a mass outflow rate of up to $\sim 1000 M_{\odot} \mathrm{yr}^{-1}$. They also inferred that the large-scale outflow was energy conserving, similar to the recent example of the nearby QSO IRAS F11119+3257 (Tombesi et al. 2015), with the outflow receiving a momentum boost by a factor of several tens, although this boost may in fact be lower (Nardini \& Zubovas 2018). Another intriguing example is the nearby QSO PG 1126-041 $(z=0.06)$, where an X-ray outflow was detected with a velocity of $-16,500 \mathrm{~km} \mathrm{~s}^{-1}$, higher than the corresponding UV mini-BAL system (Giustini et al. 2011). Similar to PDS 456, the ionized X-ray absorber was found to be highly variable.

PDS 456 appears to show many of the characteristics of the BAL QSOs. The outflow velocity of PDS 456 is very similar to the high-redshift BAL QSOs noted above. PDS 456 is also relatively X-ray quiet, and its UV-to-X-ray spectral index is steep. From the 2017 SED reported above, we find $\alpha_{\text {ox }}=$ $2.0 \pm 0.1$, as measured between the rest-frame $2500 \AA$ and $2 \mathrm{keV}$ bands (Wilkes \& Elvis 1987). This is much steeper than the mean $\alpha_{\mathrm{ox}}$ measured in radio-quiet QSOs, where, for instance, Lusso \& Risaliti (2016) find a mean value of $\left\langle\alpha_{\text {ox }}\right\rangle=1.63 \pm 0.01$ from their systematic QSO study.

The steep $\alpha_{\mathrm{ox}}$ in PDS 456 is in line with what is often observed in BAL QSOs. These are known to be X-ray quiet and can often have $\alpha_{\text {ox }}$ values around 2 or even steeper (Gallagher et al. 2006). This may be as a result of their high level of soft X-ray obscuration or from the QSOs being intrinsically X-ray weak. The soft X-ray spectrum observed here is obscured by a column of at least $N_{\mathrm{H}}=6 \times 10^{22} \mathrm{~cm}^{-2}$, and thus substantial lower-ionization gas is present in the wind in addition to the highly ionized iron $\mathrm{K}$ absorption. The steep SED of PDS 456, as parameterized through its large $\alpha_{\text {ox }}$ and from its steep $X$-ray photon index of $\Gamma=2.3$, may aide in preventing the absorbing gas from becoming overly ionized by a strong hard X-ray continuum. Such a scenario was also recently discussed by Giustini et al. (2018), who argue that AGNs with high black hole masses accreting at a high fraction of the Eddington limit are most likely to sustain a persistent disk wind. Here, the relative weakness of the X-ray to UV continuum is favorable for supporting a line-driven wind and suppressing the ionization of the gas.

Fast X-ray winds may also be present in other high accretion rate AGNs, but with smaller black hole masses, such as in the narrow-line Seyfert 1s (NLS1s). These AGNs also have steep X-ray spectra, with weak hard X-ray emission. Recently, a fast $0.25 c$ outflow was detected in the nearby NLS1 IRAS 13224-3809, from the detections of a blueshifted iron K absorption line (Parker et al. 2017) and weaker blueshifted lines in the soft X-ray band (Pinto et al. 2018). Similarly, Hagino et al. (2016) were able to model the large spectral drop above $7 \mathrm{keV}$ in the extreme NLS1 1H 0707-495 with an accretion disk wind profile, as was originally suggested by Done et al. (2007) for this AGN. Both of these AGNs also show broad blueshifted high-excitation lines in their UV spectra, most notably from C IV (Leighly \& Moore 2004), which likely has a wind origin. The C IV emission profile of PDS 456 is similar or somewhat larger, where the line centroid is shifted by $-5000 \mathrm{~km} \mathrm{~s}^{-1}$ when compared to the expected rest wavelength (O'Brien et al. 2005). For all of these AGNs, a high accretion rate with respect to Eddington is most likely the fundamental parameter for driving and sustaining a fast accretion disk wind.

\section{Conclusions}

We presented simultaneous XMM-Newton, NuSTAR, and HST observations of PDS 456, observed in 2017 March. The quasar was observed with a low X-ray flux, only matched by an earlier 2013 Suzaku observation (Matzeu et al. 2016), with a high level of X-ray obscuration. In addition to the fast $(0.25 c$, $0.4 c$ ) zones present at iron $\mathrm{K}$, in the XMM-Newton observations the soft X-ray spectrum was obscured by a lower-ionization outflowing zone of gas, with a column density of $N_{\mathrm{H}}=6 \times 10^{22} \mathrm{~cm}^{-2}$. We found that the covering fraction was variable, reaching a maximum value consistent with $100 \%$ covering during the pronounced dip in the X-ray light curve seen in OBS 1 , while the covering then declined as the source brightened thereafter.

Overall, the wind in PDS 456 is likely to be clumpy or stratified, with the wind ionization ranging by 3 orders of magnitude from the soft X-ray $(\log \xi=3)$ up to the fastest iron $\mathrm{K}$ zone $(\log \xi=6)$. The soft X-ray gas may be be located farther out, at typical distance scales of $\sim 0.1 \mathrm{pc}$, compared to the highly ionized iron $\mathrm{K}$ absorber, where the latter more likely originates from closer to the black hole nearer to the wind launching point. This gas is likely to be clumpy, and variations in the absorber covering fraction may explain the spectral 
variability, both within these observations and with earlier observations of PDS 456, which were brighter and less absorbed (Reeves et al. 2009; Nardini et al. 2015).

Potentially the soft X-ray absorber could impart measurable features onto the UV spectrum of PDS 456, through highexcitation lines such as C IV or NV. However, despite the strong X-ray obscuration, no significant UV absorption features were present in the 2017 HST spectrum, compared to the strong blueshifted absorption trough present in the original 2000 HST observation. As was discussed in Section 5.3, this may be due to the relatively high ionization of the soft X-ray absorber in PDS 456. Further simultaneous XMM-Newton and HST observations will be able to test whether or not the X-ray and UV absorption is concurrent. Nonetheless, PDS 456 bares many of the characteristics of the classical BAL quasars, and its steep SED and its high accretion rate are likely to be conducive for supporting a fast accretion disk wind.

J.R. acknowledges financial support through grants NNX17AC38G, NNX17AD56G, and HST-GO-14477.001-A. V.B. acknowledges support from the Italian Space Agency (contracts ASI-INAF I/037/12/0 and ASI-INAF n.2017-14-H.0). A.L. acknowledges support via the STFC consolidated grant ST/ K001000/1. E.N. is funded by the EU Horizon 2020 Marie Sklodowska-Curie grant no. 664931. Based on observations obtained with XMM-Newton, an ESA science mission with instruments and contributions directly funded by ESA Member States and NASA.

\section{ORCID iDs}

J. N. Reeves (16 https://orcid.org/0000-0003-3221-6765

V. Braito (1) https://orcid.org/0000-0002-2629-4989

E. Nardini (i) https://orcid.org/0000-0001-9226-8992

G. Chartas (i) https://orcid.org/0000-0003-1697-6596

A. P. Lobban (1) https://orcid.org/0000-0002-6433-1357

T. J. Turner (1) https://orcid.org/0000-0003-2971-1722

\section{References}

Behar, E., Kaspi, S., Reeves, J., et al. 2010, ApJ, 712, 26

Braito, V., Reeves, J. N., Gofford, J., et al. 2014, ApJ, 795, 87

Braito, V., Reeves, J. N., Matzeu, G. A., et al. 2018, MNRAS, 479, 3592

Cardelli, J. A., Clayton, G. C., \& Mathis, J. S. 1989, ApJ, 345, 245

Chartas, G., Brandt, W. N., \& Gallagher, S. C. 2003, ApJ, 595, 85

Chartas, G., Brandt, W. N., Gallagher, S. C., \& Garmire, G. P. 2002, ApJ, 579,169

Chartas, G., Eracleous, M., Dai, X., Agol, E., \& Gallagher, S. 2007, ApJ, 661,678

Chartas, G., Hamann, F., Eracleous, M., et al. 2014, ApJ, 783, 57

Chartas, G., Saez, C., Brandt, W. N., Giustini, M., \& Garmire, G. P. 2009, ApJ, 706, 644

Cicone, C., Maiolino, R., Sturm, E., et al. 2014, A\&A, 562, A21

Danehkar, A., Nowak, M. A., Lee, J. C., et al. 2018, ApJ, 853, 165

den Herder, J. W., Brinkman, A. C., Kahn, S. M., et al. 2001, A\&A, 365, L7

Done, C., Sobolewska, M. A., Gierliński, M., \& Schurch, N. J. 2007, MNRAS, 374, L15

Ferland, G. J., Korista, K. T., Verner, D. A., et al. 1998, PASP, 110, 761

Ferrarese, L., \& Merritt, D. 2000, ApJ, 539, 9

Feruglio, C., Ferrara, A., Bischetti, M., et al. 2017, A\&A, 608, A30

Feruglio, C., Fiore, F., Carniani, S., et al. 2015, A\&A, 583, A99
Feruglio, C., Maiolino, R., Piconcelli, E., et al. 2010, A\&A, 518, L155

Fiore, F., Feruglio, C., Shankar, F., et al. 2017, A\&A, 601, A143

Gallagher, S. C., Brandt, W. N., Chartas, G., et al. 2006, ApJ, 644, 709

Gallo, L. C., Blue, D. M., Grupe, D., Komossa, S., \& Wilkins, D. R. 2018 MNRAS, 478, 2557

Gebhardt, K. 2000, ApJ, 539, 13

Giustini, M., Cappi, M., Chartas, G., et al. 2011, A\&A, 536, A49

Giustini, M., Done, C., Proga, D., et al. 2018, A\&A, submitted

Gofford, J., Reeves, J. N., Braito, V., et al. 2014, ApJ, 784, 77

Gofford, J., Reeves, J. N., McLaughlin, D. E., et al. 2015, MNRAS, 451, 4169

Gofford, J., Reeves, J. N., Tombesi, F., et al. 2013, MNRAS, 430, 60

Grevesse, N., \& Sauval, A. J. 1998, SSRv, 85, 161

Hagino, K., Odaka, H., Done, C., et al. 2015, MNRAS, 446, 663

Hagino, K., Odaka, H., Done, C., et al. 2016, MNRAS, 461, 3954

Hamann, F., Chartas, G., McGraw, S., et al. 2013, MNRAS, 435, 133

Hamann, F., Chartas, G., Reeves, J., \& Nardini, E. 2018, MNRAS, 476, 943

Harrison, F. A., Craig, W. W., Christensen, F. E., et al. 2013, ApJ, 770, 103

Kaastra, J. S., Kriss, G. A., Cappi, M., et al. 2014, Sci, 345, 64

Kaastra, J. S., Mehdipour, M., Behar, E., et al. 2018, A\&A, submitted (arXiv:1805.03538)

Kalberla, P. M. W., Burton, W. B., Hartmann, D., et al. 2005, A\&A, 440, 775

Kallman, T. R., Palmeri, P., Bautista, M. A., Mendoza, C., \& Krolik, J. H. 2004, ApJS, 155, 675

Kriss, G. A., Lee, J. C., \& Danehkar, A. 2018a, ApJ, 859, 94

Kriss, G. A., Lee, J. C., Danehkar, A., et al. 2018b, ApJ, 853, 166

Leighly, K. M., \& Moore, J. R. 2004, ApJ, 611, 107

Longinotti, A. L., Krongold, Y., Guainazzi, M., et al. 2015, ApJL, 813, L39

Lusso, E., \& Risaliti, G. 2016, ApJ, 819, 154

Markowitz, A. G., Krumpe, M., \& Nikutta, R. 2014, MNRAS, 439, 1403

Mason, K. O., Breeveld, A., Much, R., et al. 2001, A\&A, 365, L36

Matzeu, G. A., Reeves, J. N., Braito, V., et al. 2017, MNRAS, 472, L15

Matzeu, G. A., Reeves, J. N., Nardini, E., et al. 2016, MNRAS, 458, 1311

Mehdipour, M., Kaastra, J. S., Kriss, G. A., et al. 2017, A\&A, 607, A28

Nardini, E., Reeves, J. N., Gofford, J., et al. 2015, Sci, 347, 860

Nardini, E., \& Zubovas, K. 2018, MNRAS, 478, 2274

O'Brien, P. T., Reeves, J. N., Simpson, C., \& Ward, M. J. 2005, MNRAS, 360, L25

Parker, M. L., Pinto, C., Fabian, A. C., et al. 2017, Natur, 543, 83

Parker, M. L., Reeves, J. N., Matzeu, G. A., Buisson, D. J. K., \& Fabian, A. C. 2018, MNRAS, 474, 108

Pinto, C., Alston, W., Parker, M. L., et al. 2018, MNRAS, 476, 1021

Pounds, K., Lobban, A., Reeves, J., \& Vaughan, S. 2016, MNRAS, 457, 2951

Pounds, K. A., \& Reeves, J. N. 2009, MNRAS, 397, 249

Pounds, K. A., Reeves, J. N., King, A. R., et al. 2003, MNRAS, 345, 705

Proga, D., \& Kallman, T. R. 2004, ApJ, 616, 688

Proga, D., Stone, J. M., \& Kallman, T. R. 2000, ApJ, 543, 686

Reeves, J. N., Braito, V., Gofford, J., et al. 2014, ApJ, 780, 45

Reeves, J. N., Braito, V., Nardini, E., et al. 2016, ApJ, 824, 20

Reeves, J. N., Braito, V., Nardini, E., et al. 2018a, ApJL, 854, L8

Reeves, J. N., Lobban, A., \& Pounds, K. A. 2018b, ApJ, 854, 28

Reeves, J. N., O’Brien, P. T., Braito, V., et al. 2009, ApJ, 701, 493

Reeves, J. N., O'Brien, P. T., Vaughan, S., et al. 2000, MNRAS, 312, L17

Reeves, J. N., O'Brien, P. T., \& Ward, M. J. 2003, ApJL, 593, L65

Reeves, J. N., Wynn, G., O'Brien, P. T., \& Pounds, K. A. 2002, MNRAS, 336, L56

Saez, C., \& Chartas, G. 2011, ApJ, 737, 91

Saez, C., Chartas, G., \& Brandt, W. N. 2009, ApJ, 697, 194

Sanders, D. B., Mazzarella, J. M., Kim, D.-C., Surace, J. A., \& Soifer, B. T. 2003, AJ, 126, 1607

Simpson, C., Ward, M., O’Brien, P., \& Reeves, J. 1999, MNRAS, 303, L23

Strüder, L., Briel, U., Dennerl, K., et al. 2001, A\&A, 365, L18

Tombesi, F., Cappi, M., Reeves, J. N., et al. 2010, A\&A, 521, A57

Tombesi, F., Cappi, M., Reeves, J. N., et al. 2013, MNRAS, 430, 1102

Tombesi, F., Meléndez, M., Veilleux, S., et al. 2015, Natur, 519, 436

Torres, C. A. O., Quast, G. R., Coziol, R., et al. 1997, ApJL, 488, L19

Turner, M. J. L., Abbey, A., Arnaud, M., et al. 2001, A\&A, 365, L27

Turner, T. J., Reeves, J. N., Braito, V., et al. 2018, MNRAS, 481, 2470

Veilleux, S., Meléndez, M., Sturm, E., et al. 2013, ApJ, 776, 27

Wilkes, B. J., \& Elvis, M. 1987, ApJ, 323, 243

Wilms, J., Allen, A., \& McCray, R. 2000, ApJ, 542, 914 